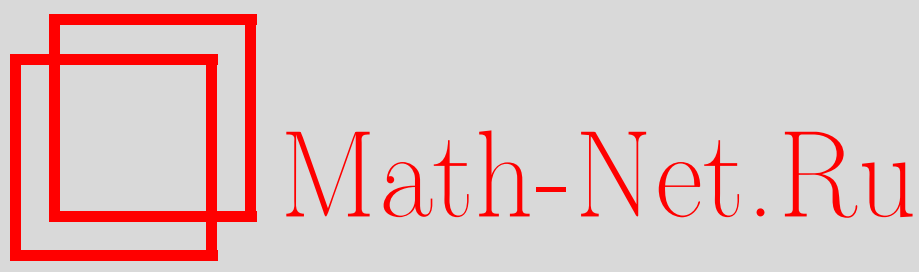

Г. Д. Степанов, Эффективные критерии знакорегулярности и осцилляционности функций Грина двухточечных краевых задач, Матем. сб., 1997, том 188, номер 11, 121-159

DOI: https://doi.org/10.4213/sm282

Использование Общероссийского математического портала Math-Net.Ru подразумевает, что вы прочитали и согласны с пользовательским соглашением

http://www.mathnet.ru/rus/agreement

Параметры загрузки:

IP : 3.89 .197 .203

26 апреля 2023 г., 15:18:11 
УДК 517.927.2

\author{
Г. Д. Степанов
}

\title{
Эффективные критерии знакорегулярности и осцилляционности функций Грина двухточечных краевых задач
}

\begin{abstract}
В статье доказываются необходимые и достаточные условия сильной знакорегулярности и осцилляционности по Гантмахеру-Крейну функции Грина двухточечной краевой задачи на собственные значения. Вьполнение этих условий даже в несамосопряженном случае обеспечивает задаче вещественность собственных значений и ряд других спектральных свойств, аналогичных тем, что присущи классической задаче Штурма-Лиувилля. Условия формулируются в терминах свойств однозначно определяемой фундаментальной системы решений дифференциального уравнения, что позволяет осуществлять их эффективную компютерную проверку и, в конечном счете, устанавливать осцилляционность функции Грина и соответствующие свойства спектра краевой задачи в тех многочисленных случаях, в которых они не обнаруживались ранее известньми достаточными признаками.

Библиографоия: 38 названий.
\end{abstract}

\section{§ 1. Введение. Формулировка и обсуждение основных результатов}

Статья посвящена обоснованию результатов, анонсированных в [1], [2]. Речь идет о необходимых и достаточных условиях сильной знакорегулярности [3] и осцилляционности [4] функции Грина краевой задачи

$$
\begin{gathered}
L x \equiv x^{(n)}+p_{1}(t) x^{(n-1)}+p_{2}(t) x^{(n-2)}+\cdots+p_{n}(t) x=\lambda r(t) x, \\
U_{i}(x) \equiv x^{\left(k_{i}\right)}(a)+\sum_{k<k_{i}} \gamma_{i k} x^{(k)}(a)=0 \quad(i=\overline{1, m}), \\
U_{i}(x) \equiv x^{\left(k_{i}\right)}(b)+\sum_{k<k_{i}} \gamma_{i k} x^{(k)}(b)=0 \quad(i=\overline{m+1, n}),
\end{gathered}
$$

где все коэффициенты вешественны, $n-1 \geqslant k_{1}>\cdots>k_{m} \geqslant 0, n-1 \geqslant k_{m+1}>$ $\cdots>k_{n} \geqslant 0,0<m<n ; r(t)$ - суммируемая неотрицательная и не эквивалентная нулю на $[a, b]$ функция, а все $p_{i}(t)$ тоже суммируемы на $[a, b]$.

Напомним, что непрерывная на квадрате $[a, b] \times[a, b]$ функция $G(t, s)$ называется знакорегулярнымм ядром интегрального уравнения

$$
x(t)=\lambda \int_{a}^{b} G(t, s) x(s) d \sigma(s),
$$

если для некоторой последовательности чисел $\varepsilon_{1}, \varepsilon_{2}, \ldots$, по модулю равных единице, при всех $p=1,2, \ldots$ выполнено условие

$$
\varepsilon_{p} G\left(\begin{array}{c}
t_{1}, t_{2}, \ldots, t_{p} \\
s_{1}, s_{2}, \ldots, s_{p}
\end{array}\right) \geqslant 0 \quad\left(a<\begin{array}{c}
t_{1}<t_{2}<\cdots<t_{p} \\
s_{1}<s_{2}<\cdots<s_{p}
\end{array}\right) .
$$


Знакорегулярное ядро называется сильно знакорегулярным, если оно дополнительно удовлетворяет следующим двум условиям

$$
\begin{gathered}
\varepsilon_{1} G(t, s)>0 \quad(a<t<b, a<s<b) \\
\varepsilon_{p} G\left(\begin{array}{c}
t_{1}, t_{2}, \ldots, t_{p} \\
t_{1}, t_{2}, \ldots, t_{p}
\end{array}\right)>0 \quad\left(a<t_{1}<\cdots<t_{p}<b, p=1,2, \ldots\right) .
\end{gathered}
$$

Если все $\varepsilon_{p}(p=1,2, \ldots)$ равны +1 , то знакорегулярное ядро называется вполне неотрицательным, а сильно знакорегулярное - осиилляционным. Сразу отметим, что для функций Грина двухточечных краевых задач знакорегулярность эквивалентна сильной знакорегулярности и возможна лишш в случае распадающихся краевых условий [3], [5], [6].

Осцилляционные и сильно знакорегулярные ядра примечательны тем, что интегральные уравнения с такими ядрами обладают цельм комплексом важных спектральных свойств. Естественно, если задача (1.1)-(1.2) имеет осцилляционную или сильно знакорегулярную функцию Грина, то соответствующий комлекс свойств автоматически распространяется и на эту задачу.

Впервые симметричные вполне неотрицательные ядра, дополнительно удовлетворяющие условию (1.5), были исследованы О. Д. Келлогом [7]-[9]. Теория осцилляционных матриц и ядер (вообше говоря, несимметричных) была развита Ф.Р. Гантмахером и М. Г. Крейном [4], [10]-[12]. Наряду с [4], [13] разнообразные аспекты, связанные со знакорегулярностью (интегральные преобразования, не повьшающие числа перемен знака, включая дискретный случай и случай суммируемых ядер, системы Чебьшёва и Маркова, интерполирование сплайнами и др.) рассматривались в циклах работ И. Шенберга и С. Карлина вместе с рядом соавторов (подробнее см. [14], [15]). В [3] результаты Гантмахера и Крейна были обобщены и дополнены (систематическое изложение относительно несимметричных ядер в [4] отсутствовало), а также была описана общая схема применения данной теории к исследованию спектральных свойств несамосопряженных краевых задач. В частности, в [3] показано, что если задача (1.1)-(1.2) имеет знакорегулярную функиию Грина, то эта функиия Грина автоматически является сильно знакорегулярной и краевая задача обладает следующими свойствами.

$1^{0}$. У задачи (1.1)-(1.2) имеется счетное множсество собственных значений $\lambda_{1}, \lambda_{2}, \ldots$, причем все они вещественные, простые $u 0<\varepsilon_{0} \varepsilon_{1} \lambda_{1}<$ $\varepsilon_{1} \varepsilon_{2} \lambda_{2}<\cdots$, где $\varepsilon_{0}=1$, а $\varepsilon_{1}, \varepsilon_{2}, \ldots$ совпадают с соответствующими величинами из (1.3).

$2^{0}$. Собственная функция $x_{i}(t)(i=1,2, \ldots)$, отвечающая собственному значению $\lambda_{i}$, имеет ровно $i-1$ нулей в $(a, b)$. Все эти нули простые, причем нули функиий $x_{i}, x_{i+1}$ перемежсаются $(i=2,3, \ldots)$. В кониах $[a, b]$ функиия $x_{i}$ имеет нули точно той кратности, которая задана краевыми условиями.

$3^{0}$. Функции $x_{1}, x_{2}, \ldots$ образуют ряд Маркова в $[a, b]$ относительно $I_{1} I_{\sigma}$, где $I_{\sigma}$ - множсество точек роста в $[a, b]$ функции $\sigma(t)=\int_{a}^{t} r(\tau) d \tau$, а $I_{1}$ интервал, получающийся исключением из $[a, b]$ точки $а$, если $k_{m}=0$, и точки $b$, ecлu $k_{n}=0$.

$4^{0}$. Всякая нетривиальная линейная комбинация $c_{k} x_{k}+\cdots+c_{r} x_{r}(k \leqslant r)$ имеет в $I_{1}$ не менее $k-1$ узловых и не более $r-1$ нулевых мест; при этом пучные нулевье места можсно засчитывать дважды. 
Таким образом, знакорегулярность функции Грина задачи (1.1)-(1.2) даже в несамосопряженном случае обеспечивает комплекс свойств, во многом аналогичных свойствам классической задачи Штурма-Лиувилля. Уже это обстоятельство (а мы совсем не затрагивали разнообразных физических приложений - см., например, [4], [16], [17]) достаточно объясняет причину интереса к признакам осцилляционности и знакорегулярности. Конечно, особый интерес представляют условия, допускающие непосредственную проверку, возможно, с помошью численных методов. Такие условия далее называются алгоритмически эффективными. Этот интуитивно ясный термин не претендует на абсолютную четкость. Важно отметить, однако, что "минорные" условия, определяющие сами понятия осцилляционности или знакорегулярности и требуюшие проверки счетного числа функциональных неравенств, весьма далеки от алгоритмической эффективности при любом разумном толковании этого термина. Остановимся на ранее известных признаках осцилляционности и знакорегулярности функций Грина несколько подробнее.

Алгоритмически эффективные критерии осцилляционности функции Грина до публикации [2] были известны лишь для частных случаев задачи (1.1)-(1.2). А именно, при $n=2$ необходимые и достаточные условия осцилляционности были получены $\Phi$. Р. Гантмахером и М. Г. Крейном в $[4$, гл. IV, $\S 10]$. Эти условия можно переф̆ормулировать в терминах свойств двух конкретных решений уравнения

$$
L x=0,
$$

в связи с чем они поддаются проверке численными методами. Кроме того, для произвольного $n$ и краевых условий вида

$$
x^{(i-1)}(a)=0(i=\overline{1, m}), \quad x^{(j-1)}(b)=0(j=\overline{1, n-m})
$$

М.Г. Крейн в [6] сформулировал два условия, каждое из которых эквивалентно осцилляционности. Первое из них - это существование у уравнения (1.6) некоторой системы решений (в [6] она определяется краевыми условиями, выписываемыми в явном виде), обладающей $W$-свойством Пойа [18]; второе эквивалентное условие - это неособенность краевой задачи и возможность представления на $(a, b)$ оператора $L$ в виде

$$
L x \equiv r_{0} \frac{d}{d t} r_{1} \frac{d}{d t} \cdots r_{n-1} \frac{d}{d t} r_{n} x,
$$

где $r_{0}(t), \ldots, r_{n}(t)$ - положительные и достаточно гладкие функции. Обоснование указанных результатов М.Г. Крейна, основанное на идее, по сушеству, присутствуюшей уже в [4], а в наиболее отчетливом виде сформулированной в [19], приведено в [3]. Здесь уместно отметить, что вообще многие результаты по данной тематике, анонсированные М. Г. Крейном, Ф.Р. Гантмахером, П. Д. Калафати, в подробном изложении авторами опубликованы не были; как нам известно из [4], $\Phi . P$. Гантмахер и М. Г. Крейн собирались посвятить несамосопряженньм задачам с осцилляционньми ядрами отдельную монографию, которая, к сожалению, так и не увидела свет.

В общем случае задача сушественно усложняется и возможность представления оператора $L$ в виде (1.8) уже не является достаточным условием осцилляционности функции Грина, хотя в любом случае остается ее необходимым условием [6].

В случае изначального задания оператора $L$ на интервале $[a, b]$ в форме $(1.8)$ и согласованного с этой факторизацией представления краевых функионалов в виде 
линейных комбинаций квазипроизводных известен достаточный признак осцилляционности функции Грина (см. $\S 5$ ), принадлежащий сразу нескольким авторам. Первый вариант формулировки и доказательства соответствующей теоремы был приведен П. Д. Калафати [20], отметившим, что аналогичньй результат одновременно с ним получен $\Phi$. Р. Гантмахером и М.Г. Крейном. Позже этот признак был заново переоткрыт (с более ясной, чем в [20], формулировкой) сначала С. Карлиным [14] и Дж. М. Кароном [21] для самосопряженных задач, а затем С. Карлиным [22] и в общем случае. Доказательства, данные в [20] и [22], используют примерно одинаковые аргументы и связаны со сложными выкладками. Более прозрачное доказательство теоремы Калафати и др. будет приведено в $\S 5$ в качестве иллюстрации общего подхода к обоснованию достаточных условий сильной знакорегулярности, детально изложенного в [3].

Упомянутый подход к обоснованию достаточных условий базируется на том, что сильная знакорегулярность функции Грина эквивалентна свойству оператора $L$ не понижать числа перемен знака на функциях, удовлетворяюших краевьм условиям (точные формулировки приведены в $\S 5$ ). Стоит отметить, что сам этот критерий получен в [3] на основе интегральных аналогов известной матричной теоремы И. Шенберга [23] с использованием ряда результатов М. Г. Крейна и его соавторов. Этот критерий не является алгоритмически эффективным, но он оказался весьма универсальньм средством доказательства достаточных признаков осцилляционности и знакорегулярности функций Грина. В [3], [24] с его помощью были получены достаточные коэффициентные условия знакорегулярности и осцилляционности для краевой задачи, заданной в обшем виде (1.1)-(1.2), выполнение которых при некоторых ограничениях на структуру краевых функционалов можно обеспечить, например, малостью интервала $[a, b]$. Соображения, близкие указанному подходу, также полезны при исследовании некоторых родственных задач (см., например, [25]-[32]). Существенную роль этот подход играет и при доказательстве основного результата настояшей работы.

Переходя к формулировке и обсуждению основных результатов статьи, условимся через $\left[z_{1}, z_{2}, \ldots, z_{i}\right](t)$ обозначать определитель Вронского, составленный из функций $z_{1}(t), z_{2}(t), \ldots, z_{i}(t)$. Критерий знакорегулярности функции Грина задачи (1.1)-(1.2), доказываемый в данной работе, формулируется через свойства ряда вронскианов, составляемых из функций, входящих в однозначно определяемую фундаментальную систему $\left\{v_{i}(t)\right\}_{1}^{n}$ решений уравнения (1.6). При работе с довольно громоздкими вронскианными выражениями для системы $\left\{v_{i}(t)\right\}_{1}^{n}$ будем пользоваться сокрашенными обозначениями

$$
v_{i j}(t)=\left[v_{1}, v_{2}, \ldots, v_{i}, v_{m+1}, v_{m+2}, \ldots, v_{m+j}\right](t) \quad(i=\overline{0, m} ; j=\overline{0, n-m})
$$

где нулевые индексы в $v_{0 j}$ и $v_{i 0}$ означают, что речь идет о вронскианах, не зависящих соответственно от $v_{i}(i \leqslant m)$ и от $v_{m+j}(1 \leqslant j)$; по определению $v_{00}=1$.

ТЕОРЕМА 1.1. Для того чтобъ задача (1.1)-(1.2) обладала знакорегулярной ( а значит и сильно знакорегулярной) функичей Грина, необходимо и достаточно, чтобь у уравнения (1.6) существовала фундаментальная система решений $v_{1}(t), v_{2}(t), \ldots, v_{n}(t)$, удовлетворяющая условиям

$$
\begin{array}{ll}
U_{i}\left(v_{j}\right)=\delta_{i j}(-1)^{n-1-k_{i}} & (i=\overline{1, n} ; j=\overline{1, m}) \\
U_{i}\left(v_{j}\right)=\delta_{i j}(-1)^{i-m} & (i=\overline{1, n} ; j=\overline{m+1, n})
\end{array}
$$


и чтобь у этой системы все вронскианьц (1.9) были отличны от нуля в $(a, b)$ и совпадали по знаку при совпадении порядков.

Если функция Грина $G(t, s)$ существует и знакорегулярна, то для указанной системы решений знаки вронскианов (1.9) порядка р совпадают со знаками характеристик $\varepsilon_{p}$ (см. (1.3)) и, кроме того, $\varepsilon_{k} \varepsilon_{k-1}=(-1)^{(n-m)} n р и$ всех $k>\min \{m, n-m\}$.

Эта теорема является частью утверждения, сформулированного в [2]. На самом деле в [2] приведены еше два условия, каждое из которых эквивалентно знакорегулярности. Сформулируем эти условия.

(А). Задача (1.1)-(1.2) обладает функиией Грина $G(t, s)$, удовлетворяющей условиям (1.3) при $p=\overline{1, n-1}$.

(Б). У уравнения (1.6) имеется линейно независимая система решений $\left\{v_{i}(t)\right\}_{1}^{n}$, удовлетворяющая условиям

$$
U_{i}\left(v_{j}\right)=U_{j}\left(v_{i}\right)=0 \quad(i=\overline{1, m} ; j=\overline{m+1, n}),
$$

такая, что все вронскианы (1.9) в $(a, b)$ отличны от нуля, совпадают по знаку при совпадении порядков и эти знаки равны вообще у всех вронскианов, порядок которых $\geqslant \min \{m, n-m\}$.

Отметим, что в частном случае $n=3$ теорема об эквивалентности знакорегулярности функции Грина (в другой терминологии) и условия, аналогичного (А), была анонсирована еще П. Д. Калафати [33], но доказательство такой эквивалентности, по-видимому, не публиковалось.

Относительно условия (Б) отметим, что имеются примеры, показывающие существенность (для обеспечения знакорегулярности) требования о совпадении знаков вронскианов, порядок которых $\geqslant \min \{m, n-m\}$. В $\S 7$ условие (Б) будет использовано в качестве промежуточного этапа при доказательстве теоремы 1.1 в части, относящейся к достаточности.

Из теоремы 1.1, в частности, следует, что, как и осцилляционность, знакорегулярность функции Грина возможна лишь в случае, когда на $(a, b)$ оператор $L$ представим в виде (1.8), поскольку отличие от нуля вронскианов (1.9) означает, что система решений $v_{i}(t)(i=1, \ldots, n)$ заведомо обладает $W$-свойством Пойа.

Говоря об алгоритмической эффективности теоремы 1.1 , отметим, что еще в [2] было указано на возможность проверки условий критерия с помощью вычислительной техники, поскольку система решений $\left\{v_{i}\right\}_{1}^{n}$ однозначно определяется условиями (1.10). В последнее время такие возможности расширились как в связи с увеличеним мощности компюютеров, так и в связи с развитием методов компютерной алгебры. Сообщение о первом варианте пакета программ анализа краевых задач на знакорегулярность в случае $L y \equiv y^{(n)}$ сделано в [34]. Уже для такого оператора соответствуюшая компьютерная программа дает интересные (в чем-то даже неожиданные) результаты и, в частности, устанавливает многочисленность случаев, в которых имеет место осцилляционность, не обнаруживаемая непосредственным применением ранее известных достаточных признаков. Так при $n=4 \mathrm{y}$ краевых задач вида

$$
\begin{aligned}
& y^{(4)}=\lambda y, \\
& y^{(3)}(0)+\alpha_{11} y^{(1)}(0)+\alpha_{10} y(0)=0, \quad y^{(3)}(1)+\beta_{11} y^{(1)}(1)+\beta_{10} y(1)=0, \\
& y^{(2)}(0)+\alpha_{21} y^{(1)}(0)+\alpha_{20} y(0)=0, \quad y^{(2)}(1)+\beta_{21} y^{(1)}(1)+\beta_{20} y(1)=0
\end{aligned}
$$


со значениями $\alpha_{i j}$ и $\beta_{i j}$ равньми \pm 1 среди 256 возможных вариантов знакорегулярность была выявлена в 55 случаях, причем ровно в 36 из них была установлена осцилляционность (например, при $\alpha_{10}=\alpha_{11}=\beta_{10}=\beta_{21}=1, \alpha_{20}=\alpha_{21}=\beta_{11}=$ $\left.\beta_{20}=-1\right)$. При непосредственном применении к задачам такого вида теоремы Калаффати-Гантмахера-Крейна-Карлина осцилляционность обнаруживается только в одном из этих случаев $\left(\alpha_{10}=\alpha_{20}=\beta_{20}=\beta_{21}=1, \alpha_{11}=\alpha_{21}=\beta_{10}=\beta_{11}=-1\right)$ и вообще не выявляется другими (известными нам) достаточными признаками.

В случае, когда речь идет именно об осцилляционности, в [1] предложены следующие необходимые и достаточные условия, имеющие внешне более простую формулировку и заметно более простое доказательство.

ТЕОРема 1.2. Для того чтобъ задача (1.1)-(1.2) обладала осциллячионной (после умножсения на $(-1)^{n-m}$ ) функиией Грина, необходимо и достаточно, чтобы у уравнения (1.6) существовала система решений $\left\{v_{i}(t)\right\}_{1}^{n}$ такая, что выполняются условия (1.11) и при $a<t<b$ все вронскианы (1.9) положительныц.

Если хотя бы в одном из концов интервала краевые условия имеют вид $x(a)=$ $\cdots=x^{(m-1)}(a)=0$ или $x(b)=\cdots=x^{(n-m-1)}(b)=0$, то оказывается, что знакорегулярность заведомо эквивалентна осцилляционности (с точностью до множителя $(-1)^{n-m}$ ) и справедливы условия осцилляционности, в которых фигурируют ограничения лишш на $n$ вронскианов.

Теорема $1.3[1]$. Пусть $G(t, s)$ - функиия Грина краевой задача (1.1)-(1.2). Тогда если $k_{1}=m-1$, то для осиилляционности ядра $(-1)^{n-m} G(t, s)$ необходимо и достаточно, чтобъ у уравнения (1.6) существовала такая система решений $\left\{v_{i}(t)\right\}_{1}^{n}$, удовлетворяющая (1.11), что в $(a, b)$ полохсительны все вронскианы

$$
v_{0 j}(t) \quad(j=\overline{1, n-m}), \quad v_{i n-m}(t) \quad(i=\overline{1, m}) .
$$

Аналогично, если $k_{m+1}=n-m-1$, то для осииллячионности ядра $(-1)^{n-m} G(t, s)$ необходимо и достаточно, чтобь у уравнения (1.6) существовала такая система решений $\left\{v_{i}(t)\right\}_{1}^{n}$, удовлетворяющая $(1.11)$, что в $(a, b)$ положительны все вронскианы

$$
v_{i 0}(t) \quad(i=\overline{1, m}), \quad v_{m j}(t) \quad(j=\overline{1, n-m}) .
$$

В отличие от теоремы 1.1 условия, задаваемые теоремами 1.2 и 1.3, не являются алгоритмически эффективньми. Алгоритмически эффективным аналогом теоремы 1.3 является следуюшее утверждение, очевидным образом вытекающее из теорем 1.1 и 1.3.

СлЕДСТВИЕ 1.1. Если $k_{1}=m-1$, то задача (1.1)-(1.2) обладает осиилляционной (после умножсения на $(-1)^{n-m}$ ) функиией Грина тогда и только тогда, когда условия (1.10) определяют систему $v_{1}(t), v_{2}(t), \ldots, v_{n}(t)$ решений уравнения (1.6), для которой в $(a, b)$ положительны все выражсения

$$
(-1)^{j(n-m)} v_{0 j}(t) \quad(j=\overline{1, n-m}), \quad(-1)^{(i+1)(n-m)} v_{i n-m}(t) \quad(i=\overline{1, m}) .
$$

Аналогично, если $k_{m+1}=n-m-1$, то задача (1.1)-(1.2) обладает осциллячионной (после умножсения на $(-1)^{n-m}$ ) функиией Грина тогда и только 
тогда, когда условия (1.10) определяют систему $v_{1}(t), v_{2}(t), \ldots, v_{n}(t)$ решений уравнения (1.6), для которой в $(a, b)$ положительны все выражсения

$$
(-1)^{i(n-m)} v_{i 0}(t) \quad(i=\overline{1, m}), \quad(-1)^{(m+j)(n-m)} v_{m j}(t) \quad(j=\overline{1, n-m}) .
$$

Обратим внимание, что следствие 1.1 включает уже упоминавшийся результат М.Г. Крейна из [6], охватывающий лишь случаи одновременного выполнения равенств $k_{1}=m-1, k_{m+1}=n-m-1$.

Доказательству необходимости в теоремах 1.1-1.3 посвящены $\S \S 2-4$, а доказательству достаточности $-\S \S 5-7$, с привлечением $\S 2$. Часть результатов, полученных при доказательстве этих теорем, имеет по нашему мнению и самостоятельный интерес, а подходы, использованные в настоящей работе, допускают обобщения, например, для многоточечных и для сингулярных задач. Особо хотелось бы отметить возможность получения аналогичных результатов для ядер типа функций Грина, которые характеризуются совпадением знаков ненулевых миноров одинакового порядка, начиная с некоторого (не обязательно первого) порядка. Класс задач с такими ядрами (повидимому, впервые отмеченньй в [25]) довольно широк, поскольку в него, например, попадает фактически каждая задача (1.1)-(1.2) при достаточной близости $a$ и $b$. Доказанные в статье теоремы 3.1 и 4.1 хорошо приспособлены для исследования таких ядер и позволяют сразу получить для соответсвующих краевых задач аналоги необходимости в теореме 1.1. Впрочем, круг вопросов, которые требуется рассмотреть для задач такого типа, весьма обширен. Некоторые предварительные результаты в этом направлении приведены в [35], [36].

\section{§2. Поведение вронскианов вблизи концов промежутка}

При доказательстве теорем 1.1-1.3 (это относится и к необходимости, и к достаточности) потребуется судить о поведении вронскианов (1.9) в окрестности концов интервала $[a, b]$. С этой целью в данном параграфе приведем несколько вспомогательных утверждений, позволяющих выделять главные члены разложения вронскианов в окрестностях концов интервала и в ряде случаев делать выводы о чередовании их знаков.

В формулировках и доказательствах параграфа неоднократно фигурируют некоторые определители, которые можно рассматривать как обобщения определителей Вронского. Для системы функций $\left\{z_{k}(t)\right\}_{1}^{p}$, где $p \leqslant n$ и все $z_{k}(t) \in C^{n-1}[a, b]$, в качестве таких обобщений вронскианов выступают миноры матрицы

$$
Z(t)=\left\|z_{j}^{(\mu)}(t)\right\| \quad(\mu=\overline{0, n-1} ; j=\overline{1, p})
$$

Далее для них используются обозначения

$$
z\left(\begin{array}{c}
M \\
J
\end{array}\right)(t)=\operatorname{det}\left\|z_{j_{k}}^{\left(\mu_{i}\right)}(t)\right\| \quad(i=\overline{1, q} ; k=\overline{1, q})
$$

где $t \in[a, b], 1 \leqslant q \leqslant p, M=\left\{\mu_{1}, \mu_{2}, \ldots, \mu_{q}\right\}$ - какой-либо набор допустимых порядков производных, а $J=\left\{j_{1}, j_{2}, \ldots, j_{q}\right\}$ - набор номеров функций.

Для произвольной числовой матрищы ранга $r$ первыми $k(k \leqslant r)$ линейно независимыми строками назовем $k$ линейно независимых строк, у которых сумма номеров минимальна. Очевидно, что наборы таких строк определены однозначно, с 
точностью до порядка строк, и если номера $m_{1}, \ldots, m_{r}$ первых $r$ линейно независимых строк упорядочены в порядке возрастания, то при $k<r$ номера $m_{1}, \ldots, m_{k}$ являются номерами первых $k$ линейно независимых строк.

Отсюда следует, что если при фиксированном $t$ ранг числовой матрицы $(2.1)$ равен $r$ и $\nu_{1}, \ldots, \nu_{r}\left(\nu_{1}<\cdots<\nu_{r}\right)$ - порядки производных в первых $r$ линейно независимых строках, то строки с порядками производных $\mu_{1}, \ldots, \mu_{k}(k \leqslant r)$ при условии $\mu_{1}+\cdots+\mu_{k} \leqslant \nu_{1}+\cdots+\nu_{k}$ и отличии $\mu_{1}, \ldots, \mu_{k}$ от перестановки $\nu_{1}, \ldots, \nu_{k}$ являются линейно зависимыми.

ЛЕмма 2.1. Пусть $t$ - фиксированная точка интервала $[a, b], r$ - ранг матрииъ (2.1) в әтой точке, $\nu_{1}, \ldots, \nu_{r}\left(\nu_{1}<\cdots<\nu_{r}\right)-$ порядки производньх в первых $r$ линейно независимых строках матрицы $Z(t), s=\sum_{k=1}^{r}\left(\nu_{k}+1-k\right)$ $u h \rightarrow 0(t+h \in[a, b])$. Тогда если $r=p$, mo

$$
\begin{gathered}
{\left[z_{1}, \ldots, z_{p}\right](t+h)=c z\left(\begin{array}{c}
\left\{\nu_{1}, \ldots, \nu_{p}\right\} \\
\{1, \ldots, p\}
\end{array}\right)(t) h^{s}+o\left(h^{s}\right),} \\
c=\frac{1}{\nu_{1} ! \cdots \nu_{p} !} \prod_{1 \leqslant j<i \leqslant p}\left(\nu_{i}-\nu_{j}\right)
\end{gathered}
$$

а если $r<p$, то

$$
\left[z_{1}, \ldots, z_{p}\right](t+h)=o\left(h^{s+(p-r)(2 n-p-r-1) / 2}\right)
$$

ДоказАТЕЛьство. С помощью формулы Тейлора с остаточньм членом в форме Пеано и очевидных переобозначений элементы матрицы $Z(t+h)$ при $k=\overline{1, p}$, $j=\overline{1, p}$ можно единообразно представить в виде

$$
z_{j}^{(k-1)}(t+h)=\sum_{i=1}^{n+1} c_{k i} \eta_{k i j} h^{i-k-\delta_{n+1 i}}
$$

где $\delta$-символ Кронекера, $c_{k i}=i(i-1) \cdots(i-k+1) / i$ !, а величины $\eta_{k i j}$, формально зависящие от трех индексов, при $i=\overline{1, n}$ равны $z_{j}^{(i-1)}(t)$ (реально от $k$ не зависят), а при $i=n+1$ уже сушественно зависят от $k$ и (что для нас главное) стремятся к нулю при $h \rightarrow 0$.

Тогда

$$
\left[z_{1}, \ldots, z_{p}\right](t+h)=\sum_{\substack{1 \leqslant i_{1} \leqslant n+1 \\ 1 \leqslant i_{p} \leqslant n+1}} c_{1 i_{1}} \cdots c_{p i_{p}} \Delta\left(i_{1}, \ldots, i_{p}\right) h^{s\left(i_{1}, \ldots, i_{p}\right)}
$$

где

$$
\begin{gathered}
\Delta\left(i_{1}, \ldots, i_{p}\right)=\operatorname{det}\left\|\eta_{k i_{k} j}\right\| \quad(k=\overline{1, p} ; j=\overline{1, p}) \\
s\left(i_{1}, \ldots, i_{p}\right)=\sum_{k=1}^{p}\left(i_{k}-k-\delta_{n+1 i_{k}}\right)
\end{gathered}
$$

Поскольку при $1 \leqslant i_{k} \leqslant n$ строка $\left(\eta_{k i_{k} 1}, \ldots, \eta_{k i_{k} p}\right)$ совпадает со строкой $\left(z_{1}^{\left(i_{k}-1\right)}(t), \ldots, z_{p}^{\left(i_{k}-1\right)}(t)\right)$ матрицы $Z(t)$, то с учетом замечания, сделанного перед формулировкой леммы, при $r<p$ определитель $\Delta\left(i_{1}, \ldots, i_{p}\right)$ может отличаться от 
нуля лишь в случае, если $s\left(i_{1}, \ldots, i_{p}\right) \geqslant s+\sum_{k=r+1}^{p}(n-k)$ (при этом он стремится к нулю при $h \rightarrow 0$ ), а при $r=p$ этот определитель может отличаться от нуля либо когда $s\left(i_{1}, \ldots, i_{p}\right)>s$, либо когда $s\left(i_{1}, \ldots, i_{p}\right)=s$ и $\left(i_{1}, \ldots, i_{p}\right)$ является перестановкой набора $\left(\nu_{1}+1, \ldots, \nu_{p}+1\right)$. С учетом сказанного из $(2.5)$ следует, что при $r<p$ заведомо вьполняется (2.4), а при $r=p$ выполняется (2.2), где

$$
c=\operatorname{det}\left\|c_{k \nu_{i}+1}\right\| \quad(k=\overline{1, p} ; i=\overline{1, p}) .
$$

Для завершения доказательства осталось проверить (2.3). Поскольку $c_{k \nu_{i}+1}=$ $\nu_{i}\left(\nu_{i}-1\right) \cdots\left(\nu_{i}-k+2\right) / \nu_{i} !$, то $(2.6)$ можно переписать в виде

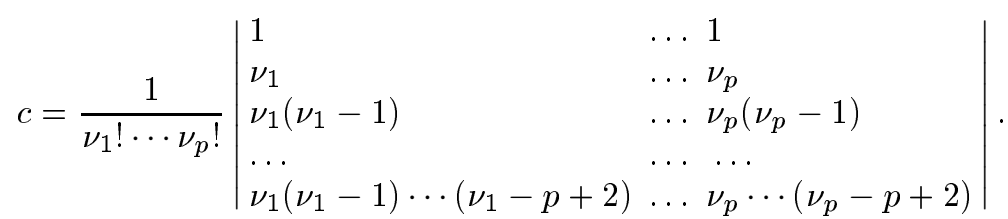

Определитель в правой части легко привести к определителю Вандермонда, добавляя к строкам (начиная с третьей) линейные комбинации предшествующих строк. Отсюда следует справедливость (2.3). Лемма доказана.

Для вронскианов вида (1.9) легко доказать следующее утверждение.

ЛЕмма 2.2. Если система функиий $\left\{v_{i}\right\}_{1}^{n}$ удовлетворяет условиям (1.10) и при некоторых $p, q, r, i_{1}, \ldots, i_{r}$ либо

$$
d=a, 1 \leqslant r \leqslant p \leqslant m, 0 \leqslant q \leqslant n-m, 1 \leqslant i_{1}<\cdots<i_{r} \leqslant p, k_{i_{1}}<p+q,
$$

либо

$d=b, 0 \leqslant p \leqslant m, 1 \leqslant r \leqslant q \leqslant n-m, m+1 \leqslant i_{1}<\cdots<i_{r} \leqslant m+q, k_{i_{1}}<p+q$,

mo

$$
v_{p q}(d)=(-1)^{\theta(d)} v\left(\begin{array}{l}
\{0, \ldots, p+q-1\} \backslash\left\{k_{i_{1}}, \ldots, k_{i_{r}}\right\} \\
\{1, \ldots, p, m+1, \ldots, m+q\} \backslash\left\{i_{1}, \ldots, i_{r}\right\}
\end{array}\right)(d)
$$

$2 \partial e$

$$
\theta(d)= \begin{cases}i_{1}+\cdots+i_{r}+r(2 n+r-1) / 2, & \text { если } d=a, \\ k_{i_{1}}+\cdots+k_{i_{r}}+r(2 p+r+1) / 2, & \text { если } d=b .\end{cases}
$$

ЗАмЕчАнИЕ. Здесь и далее знак теоретико-множественного вычитания, примененный к двум наборам номеров, означает вычеркивание в первом наборе элементов второго с сохранением порядка следования оставшихся элементов.

Опуская элементарњые выкладки, опишем схему доказательства этой леммы. Вронскиан $v_{p q}(d)$ равен определителю $(p+q) \times(p+q)$-матрицы из производных функций $v_{1}, \ldots, v_{p}, v_{m+1}, \ldots, v_{m+q}$ в точке $d$. Значение определителя этой матрицы не изменится, если при $\mu=\overline{1, r}$ строки с производными порядка $k_{i_{\mu}}$ заменить соответственно на $\left(U_{i_{\mu}}\left(v_{1}\right), \ldots, U_{i_{\mu}}\left(v_{p}\right), U_{i_{\mu}}\left(v_{m+1}\right), \ldots, U_{i_{\mu}}\left(v_{m+q}\right)\right)$, поскольку коэффициенты при ведущих производных у краевых функционалов равны 1 . В новых строках ввиду (1.10) заведомо равны нулю все элементы, кроме $U_{i_{\mu}}\left(v_{i_{\mu}}\right)$, принимающих значения \pm 1 , откуда по теореме Лапласа о детерминантах следует доказываемое утверждение.

Приведенные утверждения позволяют доказать следующую лемму о знаках вронскианов в окрестности концов интервала $[a, b]$. 
Лемма 2.3. Пусть краевая задача (1.1)-(1.2) является неособой, $\left\{v_{i}(t)\right\}_{1}^{n}-$ фундаментальная система решений уравнения $L x=0$, определяемая условиями (1.10), $0 \leqslant p \leqslant m, 0 \leqslant q \leqslant n-m, h-$ достаточно малая полодительная величина. Тогда если выполняются условия

$$
v_{p q}(a) \neq 0, \quad 0 \leqslant r<p, \quad k_{r+1}<r+q
$$

mo

$$
\operatorname{sign} v_{r q}(a+h)=(-1)^{(p-r)(n-p)} \operatorname{sign} v_{p q}(a),
$$

и, аналогично, если выполняются условия

$$
v_{p q}(b) \neq 0, \quad 0 \leqslant r<q, \quad k_{m+r+1}<r+p
$$

mo

$$
\operatorname{sign} v_{p r}(b-h)=(-1)^{(q-r) q} \operatorname{sign} v_{p q}(b) .
$$

ДоКАЗАТЕЛЬСТво. Из леммы 2.1 следует, что если выполнено (2.7), то

$$
\operatorname{sign} v_{r q}(a+h)=\operatorname{sign} v\left(\begin{array}{l}
\{0, \ldots, p+q-1\} \backslash\left\{k_{r+1}, \ldots, k_{p}\right\} \\
\{1,2, \ldots, r, m+1, m+2, \ldots, m+q\}
\end{array}\right)(a) .
$$

Отсюда и из леммы 2.2 следует справедливость (2.8).

Аналогично, в случае (2.9) из леммы 2.1 следует, что

$$
\operatorname{sign} v_{p r}(b-h)=(-1)^{s} \operatorname{sign} v\left(\begin{array}{l}
\{0, \ldots, p+q-1\} \backslash\left\{k_{m+r+1}, \ldots, k_{m+q}\right\} \\
\{1,2, \ldots, p, m+1, m+2, \ldots, m+r\}
\end{array}\right)(b),
$$

где

$$
s=(q-r)(2 p+q+r-1) / 2-\left(k_{m+r+1}+\cdots+k_{m+q}\right) .
$$

Отсюда и из леммы 2.2 следует справедливость равенства

$$
\operatorname{sign} v_{p r}(b-h)=(-1)^{s+\psi} \operatorname{sign} v_{p q}(b),
$$

где

$$
\psi=(q-r)(2 p+q-r+1) / 2+k_{m+r+1}+\cdots+k_{m+q} .
$$

Простые вычисления показывают, что четность $s+\psi$ совпадает с четностью $(q-r) q$, а значит, равенство (2.11) эквивалентно (2.10). Лемма доказана.

Лемма 2.3 означает, что для целого ряда рассматриваемых в теореме 1.1 вронскианов соотношения их знаков в окрестности концов интервала $[a, b]$ определяются только порядками ведущих производных в условиях (1.1)-(1.2) и не зависят от коэффициентов перед остальными производными. В частности, с учетом того, что вронскиан $v_{m n-m}(t)$ при всех $t$ заведомо отличен от нуля и что $v_{m 0}(a)=$ $(-1)^{m(n-1)}$ при $k_{1}=m-1, v_{0 n-m}(b)=(-1)^{(n-m)}$ при $k_{m+1}=n-m-1$, из леммы 2.3 вытекает справедливость следующего утверждения. 
СЛЕДСТВИЕ 2.1. Для вронскианов системы функций $\left\{v_{i}\right\}_{1}^{n}$, фигурирующей в теореме 1.1, при достаточно мальх полохсительных $h$ u $p=0, \ldots, m$, $q=0, \ldots, n-m$ заведомо выполняются равенства

$$
(-1)^{(m-p)(n-m)} \operatorname{sign} v_{p n-m}(a+h)=(-1)^{(n-m-q)(n-m)} \operatorname{sign} v_{m q}(b-h) .
$$

Кроме того, если в краевых условиях $k_{1}=m-1$, то

$$
\operatorname{sign} v_{p 0}(a+h)=(-1)^{p(n-m)} \quad(p=\overline{0, m}),
$$

$u$, аналогично, если $k_{m+1}=n-m-1$, mо

$$
\operatorname{sign} v_{0 q}(b-h)=(-1)^{q(n-m)} \quad(q=\overline{0, n-m})
$$

Этот результат позволяет в случае знакорегулярности функции Грина сделать вывод о чередовании знаков ряда ее миноров, что в свою очередь, как уже сказано в $\S 1$, позволяет судить о знаках соответствующих собственных значений краевой задачи. Отметим также, что доказанные свойства вронскианов полезны и при применении теоремы 1.1 для анализа конкретных краевых задач на знакорегуляность функции Грина с помощью численных методов, так как дают априорную информацию о знаках вронскианов в окрестностях концов, где некоторые из этих вронскианов аннулируются (т.е. дают именно ту информацию, получение которой численными методами вызывает затруднение).

\section{§ 3. Свойства систем функций со знакопостоянными минорами}

Доказательство необходимости в теоремах 1.1-1.3 требует обоснования $W$-свойства Пойа для определенных систем решений уравнения (1.6), в связи с чем в данном параграфе будут рассмотрены некоторые вопросы, относящиеся к теории чебьшевских и марковских систем. В частности, будет доказано следуюшее утверждение.

ТЕОРема 3.1. Пусть для системы функиий $\left\{z_{i}(t)\right\}_{1}^{n}$ из $C^{n-1}[a, b]$ при некотором $p<n$ выполнень условия

$$
\begin{gathered}
z\left(\begin{array}{c}
t_{1}, t_{2}, \ldots, t_{p} \\
1,2, \ldots, p
\end{array}\right) \geqslant 0 \quad\left(a<t_{1}<\cdots<t_{p}<b\right) \\
{\left[z_{1}, z_{2}, \ldots, z_{p+1}\right](t)>0 \quad(a<t<b)}
\end{gathered}
$$

Tогда

$$
\left[z_{1}, z_{2}, \ldots, z_{p}\right](t)>0 \quad(a<t<b) .
$$

В свою очередь, для обоснования теоремы 3.1 нам потребуется установить ряд свойств обобщенных миноров системы функций $\left\{z_{i}(t)\right\}_{1}^{n}$ (далее без дополнительных оговорок считаем, что все $\left.z_{i}(t) \in C^{n-1}[a, b]\right)$, для чего сначала приведем соответствующие определения и обозначения.

Рассматривая при некотором $p(1 \leqslant p \leqslant n)$ какой-нибудь набор номеров функций

$$
J=\left(j_{1}, j_{2}, \ldots, j_{p}\right) \quad\left(1 \leqslant j_{k} \leqslant n, k=\overline{1, p}\right)
$$


и набор $р$ пар

$$
S=\left(\left(t_{1}, i_{1}\right),\left(t_{2}, i_{2}\right), \ldots,\left(t_{p}, i_{p}\right)\right) \quad\left(a \leqslant t_{k} \leqslant b, 0 \leqslant i_{k} \leqslant n-1, k=\overline{1, p}\right)
$$

каждая из которых включает точку интервала $[a, b]$ вместе с порядком производной функций в этой точке, обозначим через

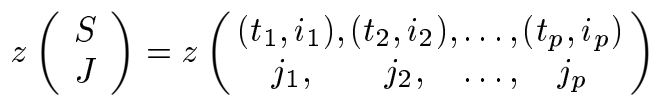

определитель матрицы

$$
\left\|z_{j_{\nu}}^{\left(i_{k}\right)}\left(t_{k}\right)\right\| \quad(k=\overline{1, p} ; \nu=\overline{1, p}) .
$$

Для произвольного набора точек $t_{1}, \ldots, t_{p}$ из $[a, b]$ при $k=\overline{1, p}$ обозначим через $r_{k}=r_{k}\left(t_{1}, \ldots, t_{k}\right)$ количество элементов набора, которые равны $t_{k}$, но имеют индексы меньшие $k$, и назовем эту величину рангом повторяемости $t_{k}$. При $a \leqslant t_{1} \leqslant \cdots \leqslant t_{p} \leqslant b, 1 \leqslant j_{1}<\cdots<j_{p} \leqslant n$ определители

$$
z^{*}\left(\begin{array}{c}
t_{1}, t_{2}, \ldots, t_{p} \\
j_{1}, j_{2}, \ldots, j_{p}
\end{array}\right)=z\left(\begin{array}{cccc}
\left(t_{1}, r_{1}\right),\left(t_{2}, r_{2}\right), \ldots, & \left(t_{p}, r_{p}\right) \\
j_{1}, & j_{2}, & \ldots, & j_{p}
\end{array}\right)
$$

называются обобщенными минорами.

Очевидно, что обобщенные миноры в отличие от определителей (3.4) не являются, вообше говоря, непрерывными функциями аргументов $t_{1}, \ldots, t_{p}$. Тем не менее они обладают важным для наших рассуждений свойством сохранять строгий знак при малых возмущениях аргументов. Точной формулировке и доказательству этого свойства обобщенных миноров предпошлем две вспомогательные леммы о средних значениях определителей (3.4).

ЛЕмма 3.1. Пусть $J=(1,2, \ldots, n)$ и для набора пар

$$
S=\left(\left(t_{1}, i_{1}\right),\left(t_{2}, i_{2}\right), \ldots,\left(t_{n}, i_{n}\right)\right)
$$

при некоторых $k$ и $m(k<m)$ выполненьи условия $t_{k}<t_{m}, i_{m}=i_{k}<$ $n-1$. Тогда существует точка $\theta \in\left(t_{k}, t_{m}\right)$ такая, что для набора $S^{*}$, получающегося из $S$ заменой пары $\left(t_{m}, i_{m}\right)$ на пару $\left(\theta, i_{m}+1\right)$, выполнено равенство

$$
z\left(\begin{array}{c}
S \\
J
\end{array}\right)=\left(t_{m}-t_{k}\right) z\left(\begin{array}{c}
S^{*} \\
J
\end{array}\right)
$$

ДоказАТЕльство. Обозначим через $\varphi(t)$ функцию переменной $t$, получающуюся заменой в

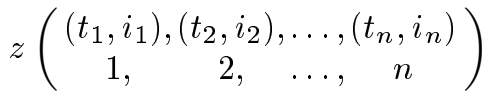

пары $\left(t_{m}, i_{m}\right)$ на $\left(t, i_{m}\right)$. Тогда $\varphi\left(t_{k}\right)=0$, а значит, сушествует $\theta \in\left(t_{k}, t_{m}\right)$ такая, что $\varphi\left(t_{m}\right)=\left(t_{m}-t_{k}\right) \varphi^{\prime}(\theta)$, что, очевидно, эквивалентно (3.6). Лемма доказана. 
Лемма 3.2. Пусть $J=(1,2, \ldots, n)$, а у набора пар (3.5) для некоторых $p$ u $q(1 \leqslant p<q \leqslant n)$ все $i_{m}=r_{m}\left(t_{1}, \ldots, t_{m}\right)$ nри $m=\overline{p, q} u$

$$
a \leqslant t_{1} \leqslant \cdots \leqslant t_{p-1}<t_{p} \leqslant t_{p+1} \leqslant \cdots \leqslant t_{q}<t_{q+1} \leqslant \cdots \leqslant t_{n} \leqslant b .
$$

Тогда существуют точки $\theta_{p}, \theta_{p+1}, \ldots, \theta_{q}$ такие, что

$$
t_{p} \leqslant \theta_{p} \leqslant \theta_{p+1} \leqslant \cdots \leqslant \theta_{q} \leqslant t_{q},
$$

и для набора пар

$$
S^{*}=\left(\left(t_{1}, i_{1}\right), \ldots,\left(t_{p-1}, i_{p-1}\right),\left(\theta_{p}, 0\right), \ldots,\left(\theta_{q}, q-p\right),\left(t_{q+1}, i_{q+1}\right), \ldots,\left(t_{n}, i_{n}\right)\right)
$$

выполнено равенство

$$
\operatorname{sign} z\left(\begin{array}{c}
S^{*} \\
J
\end{array}\right)=\operatorname{sign} z\left(\begin{array}{c}
S \\
J
\end{array}\right) .
$$

ДоКАЗАТЕЛЬСТВо. С помощью многократного применения в определенном порядке леммы 3.1 покажем сушествование конечной цепочки $S_{1}, S_{2}, \ldots, S_{N}\left(=S^{*}\right)$ наборов пар вида

$$
S_{k}=\left(\left(t_{1}, i_{1}\right), \ldots,\left(t_{p-1}, i_{p-1}\right),\left(t_{k p}, i_{k p}\right), \ldots,\left(t_{k q}, i_{k q}\right),\left(t_{q+1}, i_{q+1}\right), \ldots,\left(t_{n}, i_{n}\right)\right),
$$

в которой $S_{1}=S$, т.е. $t_{1 m}=t_{m}, i_{1 m}=i_{m}(m=\overline{p, q})$, а каждьй последующий набор $S_{k+1}$ отличается от предыдушего набора $S_{k}$ ровно одной парой (с номером от $p+1$ до $q$ ), в которой порядок производной возрастает на 1 . Каждый набор $S_{k}$ будет удовлетворять условиям

$$
\begin{gathered}
t_{p}=t_{k p} \leqslant t_{k p+1} \leqslant \cdots \leqslant t_{k q} \leqslant t_{q}, \\
i_{k p}=0, \quad i_{k m}-i_{k m-1} \leqslant 1 \quad(m=\overline{p+1, q}), \\
\operatorname{sign} z\left(\begin{array}{c}
S_{k} \\
J
\end{array}\right)=\operatorname{sign} z\left(\begin{array}{c}
S \\
J
\end{array}\right) .
\end{gathered}
$$

Кроме того, если при некотором $m(p<m \leqslant q)$ выполнено неравенство

$$
\Delta=i_{k m}-i_{k m-1} \leqslant 0,
$$

то

$$
\begin{gathered}
t_{k m}>t_{k m-1}=t_{k m-2}=t_{k m-3}=\cdots=t_{k m-1+\Delta}, \\
i_{k m}=i_{k m-1}+\Delta=i_{k m-2}+\Delta+1=i_{k m-3}+\Delta+2=\cdots=i_{k m-1+\Delta} .
\end{gathered}
$$

Справедливость всех этих свойств для набора $S_{1}=S$ очевидньм образом следует из условий леммы. Далее по индукции, если $S_{k}$ удовлетворяет перечисленньм свойствам и существуют номера $m(p<m \leqslant q)$ такие, что вьполняется (3.10), то выберем в качестве $m$ наибольший из таких номеров. Тогда по лемме 3.1 ввиду $(3.11)$ и $(3.12)$ в интервале $\left(t_{k m-1}, t_{k m}\right)$ найдется точка $t_{k+1 m}$ такая, что замена в $S_{k}$ пары $\left(t_{k m}, i_{k m}\right)$ на $\left(t_{k+1 m}, i_{k m}+1\right)$ дает набор $S_{k+1}$, для которого

$$
\operatorname{sign} z\left(\begin{array}{c}
S_{k+1} \\
J
\end{array}\right)=\operatorname{sign} z\left(\begin{array}{c}
S_{k} \\
J
\end{array}\right) .
$$

Очевидно, что при такой замене справедливость соответствующих свойств (3.7) $-(3.9)$ и (3.11), (3.12), при условии $(3.10)$, сохраняется и для $S_{k+1}$. Описанный процесс замены пар продолжается, пока для некоторых $m$ выполняется (3.10), и за конечное число шагов заканчивается ввиду (3.8) при таком $k=N$, что $i_{k m}=m-p$ $(m=\overline{p, q})$. Последнее вместе с $(3.7)$ и $(3.9)$ означает, что $S_{N}$ удовлетворяет всем условиям, которые требовалось установить для $S^{*}$. Лемма доказана. 
ЛЕмма 3.3. Пусть для некоторого упорядоченного набора $t_{1}, t_{2}, \ldots, t_{n}$ $\left(a \leqslant t_{1} \leqslant t_{2} \leqslant \cdots \leqslant t_{n} \leqslant b\right)$ выполнено условие

$$
z^{*}\left(\begin{array}{c}
t_{1}, t_{2}, \ldots, t_{n} \\
1,2, \ldots, n
\end{array}\right)>0
$$

Тогда существует $\varepsilon>0$ такое, ито

$$
z^{*}\left(\begin{array}{c}
\tau_{1}, \tau_{2}, \ldots, \tau_{n} \\
1,2, \ldots, n
\end{array}\right)>0
$$

для всех $\left\{\tau_{i}\right\}_{1}^{n}$, удовлетворяющих условиям

$$
a \leqslant \tau_{1} \leqslant \tau_{2} \leqslant \cdots \leqslant \tau_{n} \leqslant b, \quad\left|\tau_{j}-t_{j}\right|<\varepsilon \quad(j=\overline{1, n}) .
$$

ДокАЗАТЕЛЬСтво. Для последовательности $\left\{t_{k}\right\}_{1}^{n}$ все индексы $k$, при которых $r_{k}=0$, в порядке возрастания обозначим через $k_{1}, k_{2}, \ldots, k_{p}$. Тогда

$$
k_{1}=1, \quad t_{k_{j}}>t_{k_{j}-1}(j=\overline{2, p})
$$

и без ограничения обшности можно считать, что $\varepsilon$-окрестности точек $t_{k_{1}}, \ldots, t_{k_{p}}$ не пересекаются друг с другом. Очевидно, что в этом случае для любого набора $\left\{\tau_{k}\right\}_{1}^{n}$, удовлетворяюшего (3.15), при тех же $k_{1}, \ldots, k_{p}$ выполнено условие

$$
\tau_{k_{j}} \neq \tau_{k_{j}-1} \quad(j=\overline{2, p})
$$

(что, конечно, не исключает наличия других индексов, при которых $\tau_{k} \neq \tau_{k-1}$ ).

По лемме 3.2 для любого такого набора $\left\{\tau_{k}\right\}_{1}^{n}$ в свою очередь найдется набор $\left\{\theta_{k}\right\}_{1}^{n}\left(a \leqslant \theta_{1} \leqslant \cdots \leqslant \theta_{n} \leqslant b\right)$ такой, что

$$
\theta_{k} \in\left(t_{k}-\varepsilon, t_{k}+\varepsilon\right) \quad(k=\overline{1, n}),
$$

и при $J=(1,2, \ldots, n)$ для набора пар

$$
S=(\overbrace{\left(\theta_{k_{1}}, 0\right),\left(\theta_{k_{1}+1}, 1\right), \ldots,}^{k_{2}-k_{1}} \overbrace{\left(\theta_{k_{2}}, 0\right),\left(\theta_{k_{2}+1}, 1\right), \ldots, \ldots,}^{k_{3}-k_{2}}, \overbrace{\left(\theta_{k_{p}}, 0\right), \ldots,\left(\theta_{n}, n-k_{p}\right)}^{n+1-k_{p}})
$$

справедливо равенство

$$
\operatorname{sign} z\left(\begin{array}{c}
S \\
J
\end{array}\right)=\operatorname{sign} z^{*}\left(\begin{array}{c}
\tau_{1}, \tau_{2}, \ldots, \tau_{n} \\
1,2, \ldots, n
\end{array}\right) .
$$

С другой стороны, ввиду (3.13) и непрерывности определителей (3.4) при достаточно малых $\varepsilon$ вьполняется неравенство

$$
\operatorname{sign} z\left(\begin{array}{c}
S \\
J
\end{array}\right)>0
$$

Поэтому при малых $\varepsilon>0$ из (3.15) обязательно следует (3.14). Лемма доказана.

Если $t_{1}=t_{2}=\cdots=t_{n}=t$, то определитель в левой части (3.13) совпадает с вронскианом $\left[z_{1}, z_{2}, \ldots, z_{n}\right](t)$. Для этого случая лемму 3.3 удобно переформулировать в следующем виде. 
СлЕДСТВИЕ 3.1. Если при некотором $t \in(a, b)$ вронскиан $\left[z_{1}, z_{2}, \ldots, z_{n}\right](t)$ положителен, то при достаточно малом $\varepsilon>0 u t-\varepsilon<t_{1} \leqslant t_{2} \leqslant \cdots \leqslant t_{n}<$ $t+\varepsilon$ все обобщенные миноры

$$
z^{*}\left(\begin{array}{c}
t_{1}, t_{2}, \ldots, t_{n} \\
1,2, \ldots, n
\end{array}\right)
$$

тоже положительнь.

Заметим, что требование малости возмушения в лемме 3.3 сушественно. Например, для $z_{1}(t)=\cos (\pi t / \varepsilon), z_{2}(t)=\sin (\pi t / \varepsilon)(\varepsilon>0)$ вронскиан $\left[z_{1}, z_{2}\right](t)=\pi / \varepsilon>0$ при всех $t$, а миноры

$$
z\left(\begin{array}{c}
t_{1}, t_{2} \\
1,2
\end{array}\right)=\sin \left(\pi\left(t_{2}-t_{1}\right) / \varepsilon\right) \quad\left(a<t_{1}<t_{2}<b\right)
$$

положительны, если $b-a<\varepsilon$, и могут принимать значения любого знака, если $b-a>\varepsilon$.

Из леммы 3.3 также, очевидным образом, вытекает требуюшееся нам в дальнейшем следуюшее хорошо известное утверждение.

СлЕДСТВИЕ 3.2. Для того чтобъ при $(a \leqslant) t_{1} \leqslant t_{2} \leqslant \cdots \leqslant t_{n}(\leqslant b)$ все обобщенные миноры (3.16) были неотричательнымм, необходимо и достаточно, чтобы при $а<t_{1}<t_{2}<\cdots<t_{n}<$ были неотричательными все миноры

$$
z\left(\begin{array}{c}
t_{1}, t_{2}, \ldots, t_{n} \\
1,2, \ldots, n
\end{array}\right) .
$$

В данном параграфе нам также потребуются некоторые факты о взаимосвязях обобшенных миноров, в основе которых лежат чисто матричные соотношения, формулируемые здесь в виде двух лемм для $(m \times n)$-матрицы

$$
A=\left\|a_{i j}\right\| \quad(i=\overline{1, m}, j=\overline{1, n}) .
$$

Лемма 3.4. Пусть в матрице (3.17) при некотором $p<\min \{m, n\}$ определитель

$$
A\left(\begin{array}{c}
i_{1}, i_{2}, \ldots, i_{p} \\
1,2, \ldots, p
\end{array}\right)
$$

равен нулю. Тогда при $k=\overline{1, p}, l=\overline{1, p}, i^{\prime}=\overline{1, m}, i^{\prime \prime}=\overline{1, m}$ справедливо равенство

$$
\begin{gathered}
{\left[A\left(\begin{array}{cc}
i_{1}, & i_{p} \\
1, \ldots, l-1, l+1, \ldots, p, p+1
\end{array}\right)\right]^{2} A\left(\begin{array}{cc}
i_{1}, \ldots, i_{k-1}, i_{k+1}, \ldots, i_{p}, i^{\prime} \\
1,
\end{array}\right)} \\
\times A\left(\begin{array}{c}
i_{1}, \ldots, i_{k-1}, i_{k+1}, \ldots, i_{p}, i^{\prime \prime} \\
1, \\
\ldots
\end{array}\right) \\
=\left[A\left(\begin{array}{c}
i_{1}, \ldots, i_{k-1}, i_{k+1}, \ldots, i_{p} \\
1, \ldots, l-1, l+1, \ldots, p
\end{array}\right)\right]^{2} \\
\quad \times A\left(\begin{array}{c}
i_{1}, \ldots, i_{p}, i^{\prime} \\
1, \ldots, p, p+1
\end{array}\right) A\left(\begin{array}{c}
i_{1}, \ldots, i_{p}, i^{\prime \prime} \\
1, \ldots, p, p+1
\end{array}\right) .
\end{gathered}
$$

$B$ случае $p=1$ считается, что $A\left(\begin{array}{c}i_{1}, \ldots, i_{k-1}, i_{k+1}, \ldots, i_{p} \\ 1, \ldots, l-1, l+1, \ldots, p\end{array}\right) \equiv 1$. 
ДоказАТЕЛЬство. Сразу отметим, что если в наборе $i_{1}, \ldots, i_{p}$ есть совпадающие элементы, то равенство (3.18) тривиально, а если совпадающих элементов нет, то с помощью перестановок строк и столбцов рассматриваемый общий случай очевидньм образом сводится к случаю, когда $i_{q}=q(q=\overline{1, p}), k=p, l=p$. Для обоснования леммы в этом случае воспользуемся известньм детерминантным тождеством Сильвестра (см. [37])

$$
\left|\begin{array}{ll}
A\left(\begin{array}{l}
1, \ldots, p-1, p \\
1, \ldots, p-1, p
\end{array}\right) & A\left(\begin{array}{l}
1, \ldots, p-1, p \\
1, \ldots, p-1, j
\end{array}\right) \\
A\left(\begin{array}{l}
1, \ldots, p-1, i \\
1, \ldots, p-1, p
\end{array}\right) & A\left(\begin{array}{l}
1, \ldots, p-1, i \\
1, \ldots, p-1, j
\end{array}\right)
\end{array}\right|=A\left(\begin{array}{c}
1, \ldots, p-1 \\
1, \ldots, p-1
\end{array}\right) A\left(\begin{array}{l}
1, \ldots, p, i \\
1, \ldots, p, j
\end{array}\right),
$$

которое справедливо при всех $i$ и $j$. Раскрывая определитель в левой части, с учетом условия леммы получаем равенство

$$
-A\left(\begin{array}{c}
1, \ldots, p-1, p \\
1, \ldots, p-1, j
\end{array}\right) A\left(\begin{array}{c}
1, \ldots, p-1, i \\
1, \ldots, p-1, p
\end{array}\right)=A\left(\begin{array}{c}
1, \ldots, p-1 \\
1, \ldots, p-1
\end{array}\right) A\left(\begin{array}{l}
1, \ldots, p, i \\
1, \ldots, p, j
\end{array}\right) .
$$

Заменяя здесь поочередно $i$ на $i^{\prime}$ и на $i^{\prime \prime}$, а $j$ на $p+1$, приходим к двум аналогичньм равенствам, почленное перемножение которых дает проверяемое соотношение

$$
\begin{aligned}
& {\left[A\left(\begin{array}{l}
1, \ldots, p-1, \quad p \\
1, \ldots, p-1, p+1
\end{array}\right)\right]^{2} A\left(\begin{array}{l}
1, \ldots, p-1, i^{\prime} \\
1, \ldots, p-1, p
\end{array}\right) A\left(\begin{array}{l}
1, \ldots, p-1, i^{\prime \prime} \\
1, \ldots, p-1, p
\end{array}\right)} \\
& =\left[A\left(\begin{array}{l}
1, \ldots, p-1 \\
1, \ldots, p-1
\end{array}\right)\right]^{2} A\left(\begin{array}{l}
1, \ldots, p, i^{\prime} \\
1, \ldots, p, p+1
\end{array}\right) A\left(\begin{array}{l}
1, \ldots, p, i^{\prime \prime} \\
1, \ldots, p, p+1
\end{array}\right) .
\end{aligned}
$$

Лемма доказана.

Лемма 3.5. Пусть у матрицы (3.17) все миноры р-го поряджа, расположсенные в первых р столбцах, неотрицательны, а все миноры $(p+1)$-го порядка, расположенные в первых $p+1$ столбцах, строго положительны. Тогда при

$$
1<i_{1}<i_{2}<\cdots<i_{p}<m
$$

минорь

$$
A\left(\begin{array}{c}
i_{1}, i_{2}, \ldots, i_{p} \\
1,2, \ldots, p
\end{array}\right)
$$

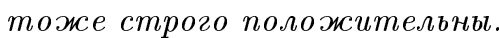

ДоКАЗАТЕЛЬСТво проведем методом от противного. Предположим, что условия леммы выполнены, но при некотором наборе $i_{1}, \ldots, i_{p}$, удовлетворяюшем (3.19), минор (3.20) равен нулю. Тогда по лемме 3.4 при $i^{\prime}=1, i^{\prime \prime}=m$ и всех $k=\overline{1, p}$, $l=\overline{1, p}$ вьполняется равенство (3.18). В этом случае, с учетом условия доказываемой леммы, в (3.18) один из определителей

$$
A\left(\begin{array}{cr}
i_{1}, \ldots, i_{k-1}, i_{k+1}, \ldots, i_{p}, i^{\prime} \\
1, & \ldots,
\end{array}\right), \quad A\left(\begin{array}{c}
i_{1}, \ldots, i_{p}, i^{\prime} \\
1, \ldots, p, p+1
\end{array}\right)
$$

должен быть неположительным, а все остальные сомножители в левой и правой частях (3.18) неотрицательны. Следовательно, и левая, и правая части равны 
нулю. Поскольку все миноры $(p+1)$-го порядка строго положительны, то при всех $k=\overline{1, p}, l=\overline{1, p}$ должны равняться нулю определители

$$
A\left(\begin{array}{c}
i_{1}, \ldots, i_{k-1}, i_{k+1}, \ldots, i_{p} \\
1, \ldots, l-1, l+1, \ldots, p
\end{array}\right) .
$$

При $p=1$ этого не может быть ввиду замечания к лемме 3.4 , а при $p>1$ это влекло бы равенство нулю минора

$$
A\left(\begin{array}{c}
i_{1}, \ldots, i_{p}, m \\
1, \ldots p, p+1
\end{array}\right) .
$$

Таким образом, предположение о равенстве нулю определителя (3.20) приводит к противоречию, которое завершает доказательство леммы.

Лемма 3.5, очевидно, останется справедливой, если в ее формулировке матрицу заменить на систему функций, а миноры матрищы заменить на миноры системы функций. Покажем, что такое утверждение справедливо и в случае использования обобщенных миноров. Получающийся при этом результат родственен по смыслу теореме 3.1 и является важньм этапом в доказательстве этой теоремы.

Лемма 3.6. Пусть у системы функиий $\left\{z_{i}(t)\right\}_{1}^{n}$ при некотором $p(1 \leqslant p<n)$ все определители

$$
z^{*}\left(\begin{array}{c}
t_{1}, t_{2}, \ldots, t_{p} \\
1,2, \ldots, p
\end{array}\right)
$$

неотрицательны при

$$
a<t_{1} \leqslant t_{2} \leqslant \cdots \leqslant t_{p}<b
$$

а все определители

$$
z^{*}\left(\begin{array}{c}
t_{1}, t_{2}, \ldots, t_{p+1} \\
1,2, \ldots, p+1
\end{array}\right)
$$

строго положительны при $a<t_{1} \leqslant t_{2} \leqslant \cdots \leqslant t_{p+1}<b$. Тогда все определители (3.21) при условии (3.22) тоже строго полохсительны.

ДокАЗАтЕльство. Строгая положительность определителей (3.21), если $a<t_{1}<t_{2}<\cdots<t_{p}<b$, следует непосредственно из леммы 3.5. Поэтому, чтобы доказать справедливость леммы в общем случае достаточно показать, что из существования набора $\left\{t_{i}\right\}_{1}^{p}$ точек из $(a, b)$, для которого определитель $(3.21)$ равен нулю, следовало бы существование аналогичного набора, имеющего строго меньшее число кратных точек.

Итак, предположим, что существует набор $\left\{t_{i}\right\}_{1}^{p}$, удовлетворяющий $(3.22)$ и включающий кратные точки, для которого определитель (3.21) равен нулю. Заметим, что в этом случае при

$$
1 \leqslant k \leqslant p, \quad 1 \leqslant l \leqslant p, a<t^{\prime}<t_{1}, t_{p}<t^{\prime \prime}<b
$$

справедливо равенство

$$
\begin{aligned}
& {\left[z^{*}\left(\begin{array}{l}
t_{1}, \ldots, t_{p} \\
1, \ldots, l-1, l+1, \ldots, p, p+1
\end{array}\right)\right]^{2}} \\
& \quad \times z^{*}\left(\begin{array}{c}
t_{1}, \ldots, t_{k-1}, t_{k+1}, \ldots, t_{p}, t^{\prime} \\
1, \quad, p
\end{array}\right) z^{*}\left(\begin{array}{c}
t_{1}, \ldots, t_{k-1}, t_{k+1}, \ldots, t_{p}, t^{\prime \prime} \\
1, \\
\ldots, p
\end{array}\right) \\
& =\left[z^{*}\left(\begin{array}{c}
t_{1}, \ldots, t_{k-1}, t_{k+1}, \ldots, t_{p} \\
1, \ldots l-1-1, l+1 \ldots, p
\end{array}\right)\right]^{2} \\
& \quad \times z^{*}\left(\begin{array}{l}
t_{1}, \ldots, t_{p}, t^{\prime} \\
1, \ldots, p, p+1
\end{array}\right) z^{*}\left(\begin{array}{c}
t_{1}, \ldots, t_{p}, t^{\prime \prime} \\
1, \ldots, p, p+1
\end{array}\right) .
\end{aligned}
$$


Действительно, если $t_{k}<t_{k+1}$ или $k=p$, то справедливость (3.25) непосредственно следует из леммы 3.4 , примененной к матрице

$$
\left(\begin{array}{llll}
z_{1}^{\left(r_{1}\right)}\left(t_{1}\right) & z_{2}^{\left(r_{1}\right)}\left(t_{1}\right) & \ldots & z_{p+1}^{\left(r_{1}\right)}\left(t_{1}\right) \\
z_{1}^{\left(r_{2}\right)}\left(t_{2}\right) & z_{2}^{\left(r_{2}\right)}\left(t_{2}\right) & \ldots & z_{p+1}^{\left(r_{2}\right)}\left(t_{2}\right) \\
\ldots \ldots \ldots \ldots & \ldots \ldots & \ldots \ldots & \ldots \ldots \\
z_{1}^{\left(r_{p}\right)}\left(t_{p}\right) & z_{2}^{\left(r_{p}\right)}\left(t_{p}\right) & \ldots & z_{p+1}^{\left(r_{p}\right)}\left(t_{p}\right) \\
z_{1}\left(t^{\prime}\right) & z_{2}\left(t^{\prime}\right) & \ldots & z_{p+1}\left(t^{\prime}\right) \\
z_{1}\left(t^{\prime \prime}\right) & z_{2}\left(t^{\prime \prime}\right) & \ldots & z_{p+1}\left(t^{\prime \prime}\right)
\end{array}\right)
$$

где $r_{1}, r_{2}, \ldots, r_{p}$ - ранги повторяемости элементов набора $t_{1}, t_{2}, \ldots, t_{p}$. Случай же, когда $t_{k}=t_{k+1}$, фактически эквивалентен уже рассмотренному, так как если $t_{k}=t_{k+1}=\cdots=t_{q}$, то

$$
\begin{aligned}
z^{*}\left(\begin{array}{c}
t_{1}, \ldots, t_{k-1}, t_{k+1}, \ldots, t_{p}, t \\
1, \ldots,
\end{array}\right) & =z^{*}\left(\begin{array}{c}
t_{1}, \ldots, t_{q-1}, t_{q+1}, \ldots, t_{p}, t \\
1, \ldots, \\
1,
\end{array}\right), \\
z^{*}\left(\begin{array}{c}
t_{1}, \ldots, t_{k-1}, t_{k+1}, \ldots, t_{p} \\
1, \ldots, l-1, l+1, \ldots, p
\end{array}\right) & =z^{*}\left(\begin{array}{c}
t_{1}, \ldots, t_{q-1}, t_{q+1}, \ldots, t_{p} \\
1, \ldots, l-1, l+1, \ldots, p
\end{array}\right) .
\end{aligned}
$$

При тех же условиях (3.24) левая и правая части равенства (3.25) не могут отличаться от нуля, так как в таком случае с учетом условий доказываемой леммы они имели бы противоположные знаки. Но правая часть (3.25) может обрашаться в нуль только за счет первого сомножителя, так как все определители вида (3.23) строго положительны. Таким образом, при всех $k=\overline{1, p}, l=\overline{1, p}$ определители

$$
z^{*}\left(\begin{array}{c}
t_{1}, \ldots, t_{k-1}, t_{k+1}, \ldots, t_{p} \\
1, \ldots, l-1, l+1, \ldots, p
\end{array}\right)
$$

равны нулю. Отсюда следует, что при замене в наборе $\left\{t_{i}\right\}_{1}^{p}$ любой кратной точки на $t^{\prime \prime}\left(t_{p}<t^{\prime \prime}<b\right)$, равенство нулю соответствующего определителя $p$-го порядка сохранится, а количество кратных точек в наборе уменьшится, что завершает доказательство леммы.

Теперь докажем сформулированную в начале данного параграфа теорему 3.1. В силу следствия 3.1 из (3.2) следует, что для любой точки $t \in(a, b)$ найдется такое $\varepsilon>0$, что при $t-\varepsilon<t_{1} \leqslant \cdots \leqslant t_{p+1}<t+\varepsilon$ определители (3.23) строго положительны. Отсюда и из (3.1), с учетом следствия 3.2 , по лемме 3.6 следует положительность определителей (3.21) при $t-\varepsilon<t_{1} \leqslant \cdots \leqslant t_{p}<$ $t+\varepsilon$ и, в частности, справедливость неравенства $\left[z_{1}, z_{2}, \ldots, z_{p}\right](t)>0$. Ввиду произвольности $t$ это означает справедливость (3.3). Теорема 3.1 доказана.

Как было уже отмечено, вопросы рассмотренные в этом параграффе, фактически, относятся к теории чебышевских и марковских систем. В частности, из теоремы 3.1 вытекает следующий результат.

СлЕДСТВИЕ 3.3. Пусть для системь функиий $\left\{z_{i}(t)\right\}_{1}^{n}$ nри всех $p=\overline{1, n}$ выполнень условия (3.1) $u\left[z_{1}, \ldots, z_{n}\right](t)>0(a<t<b)$. Тогда рассматриваемая система в $(a, b)$ обладает $W$-свойством Пойа (а значит, согласно [15] является ЕCT-системой в $(a, b))$.

Несмотря на наличие целого ряда фундаментальных монографий по чебышевским системам, обнаружить в них соответствуюшего результата нам не удалось. 
Однако, в [13] сформулировано родственное утверждение (У.4.2), согласно которому, если система $\left\{z_{i}(t)\right\}_{1}^{n}$ является $T_{+}$-системой в $(a, b)$ и при всех $p<n$ удовлетворяет условию $(3.1)$, то она является и $M_{+}$-системой в $(a, b)(C T$-системой в терминологии [15]).

Следует также отметить, что ни теорема 3.1 , ни следствие 3.3 не допускают усиления "напрашиваюшейся" заменой условия (3.1) на условие

$$
\left[z_{1}, z_{2}, \ldots, z_{p}\right](t) \geqslant 0 \quad(a \leqslant t \leqslant b) .
$$

Так, для рассматриваемых на $[-1,1]$ функций $z_{1}(t)=t^{2}, z_{2}(t)=-t, z_{3}(t)=$ $t^{3}-5 t+4$ имеем $\left[z_{1}, z_{2}, z_{3}\right](t)=2 t^{3}+8>0$ и $\left[z_{1}\right](t)=\left[z_{1}, z_{2}\right](t)=t^{2} \geqslant 0$, т.е. на $[-1,1]$ условия (3.26) при всех $p$ и условие (3.2) при $p=n-1=2$ выполняются, а условие (3.3) нарушается в точке $t=0$. Естественно, нарушается и условие (3.1): $z\left(\begin{array}{c}t_{1}, t_{2} \\ 1,2\end{array}\right)=t_{1} t_{2}\left(t_{2}-t_{1}\right)$ при $-1<t_{1}<t_{2}<1$ меняет знак.

\section{$\S 4$. Свойства миноров функции Грина. Доказательство необходимости в теоремах 1.1-1.3}

Основная цель данного параграфа - завершение доказательства необходимости в теоремах 1.1-1.3, однако предварительно будут приведены некоторые общие факты о функции Грина краевой задачи (1.1)-(1.2).

Известно, что для сушествования функции Грина рассматриваемой задачи необходимо и достаточно, чтобы эта задача была неособой, т.е. соответствуюшая однородная задача имела только тривиальное решение $x(t)=0$. Очевидно, что неособенность задачи эквивалентна существованию у уравнения (1.6) фундаментальной системы решений, удовлетворяюшей условиям (1.11); то же относится и к условиям (1.10). Далее на протяжении всего параграфа считаем, что задача (1.1)-(1.2) является неособой, а система функций $\left\{v_{i}(t)\right\}_{1}^{n}$ является фундаментальной системой решений, определяемой условиями (1.10). Через эти решения функция Грина представима в виде

$$
G(t, s)=\left\{\begin{array}{cl}
-\sum_{i=m+1}^{n} v_{i}(t) y_{i}(s), & a \leqslant t \leqslant s \leqslant b \\
\sum_{i=1}^{m} v_{i}(t) y_{i}(s), & a \leqslant s \leqslant t \leqslant b
\end{array}\right.
$$

где

$$
y_{i}(s)=(-1)^{n+i} \frac{\left[v_{1}, \ldots, v_{i-1}, v_{i+1}, \ldots, v_{n}\right](s)}{\left[v_{1}, v_{2}, \ldots, v_{n}\right](s)} \quad(i=\overline{1, n}) .
$$

Действительно, производная $k$-го порядка по $t$ функции

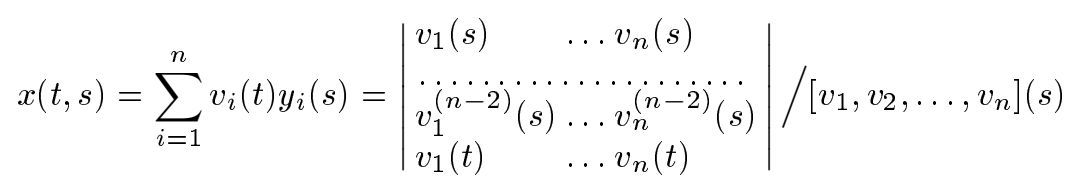

при $t=s$ равна нулю, если $k \leqslant n-2$, и равна единице, если $k=n-1$. Поэтому функция $G(t, s)$, определяемая соотношением (4.1), непрерывна и имеет непрерывные по $t$ производные до $(n-2)$-го поря дка при всех $t$ и $s$ из $[a, b]$, а при фиксированном $s$ из $[a, b]$ имеет также абсолютно непрерывную производную $(n-1)$-го порядка 
по $t$ в каждом из интервалов $[a, s)$ и $(s, b]$, которая при $t=s$ испытывает скачок, равный 1 . Кроме того, в каждом из интервалов $[a, s)$ и $(s, b]$ функция $G(t, s)$ как функция переменной $t$ удовлетворяет уравнению $L(G)=0$ (почти всюду) и краевым условиям $U_{i}(G)=0(i=\overline{1, n})$. Таким образом, $G(t, s)$ удовлетворяет всем условиям, определяющим функцию Грина.

Лемма 4.1. Пусть $r=\max \{m, n-m\}$,

$$
a \leqslant a_{1}<\cdots<a_{r}<t_{1}<\cdots<t_{n}<b_{1}<\cdots<b_{r} \leqslant b .
$$

Тогда при $p=\overline{0, m}, q=\overline{0, n-m}$ справедливь равенства

$$
\begin{aligned}
G & \left(\begin{array}{c}
t_{1}, \ldots, t_{p}, t_{p+1}, \ldots, t_{p+q} \\
a_{1}, \ldots, a_{p}, b_{1}, \ldots, b_{q}
\end{array}\right) \\
& =(-1)^{q} \sum v\left(\begin{array}{c}
t_{1}, \ldots, t_{p+q} \\
i_{1}, \ldots, i_{p+q}
\end{array}\right) y\left(\begin{array}{c}
a_{1}, \ldots, a_{p} \\
i_{1}, \ldots, i_{p}
\end{array}\right) y\left(\begin{array}{c}
b_{1}, \ldots, b_{q} \\
i_{p+1}, \ldots, i_{p+q}
\end{array}\right), \\
G & \left(\begin{array}{c}
a_{1}, \ldots, a_{q}, b_{1}, \ldots, b_{p} \\
t_{1}, \ldots, t_{p}, t_{p+1}, \ldots, t_{p+q}
\end{array}\right) \\
& =(-1)^{q} \sum v\left(\begin{array}{c}
a_{1}, \ldots, a_{q} \\
i_{p+1}, \ldots, i_{p+q}
\end{array}\right) v\left(\begin{array}{c}
b_{1}, \ldots, b_{p} \\
i_{1}, \ldots, i_{p}
\end{array}\right) y\left(\begin{array}{c}
t_{1}, \ldots, t_{p+q} \\
i_{1}, \ldots, i_{p+q}
\end{array}\right),
\end{aligned}
$$

где суммирование ведется по всем наборам $i_{1}, \ldots, i_{p+q}$ таким, что

$$
1 \leqslant i_{1}<\cdots<i_{p} \leqslant m<i_{p+1}<\cdots<i_{p+q} \leqslant n .
$$

ЗАмЕчАнИЕ. В формулировке леммы равенство $p=0$ означает отсутствие элементов в наборах $a_{1}, \ldots, a_{p}, i_{1}, \ldots, i_{p}, t_{1}, \ldots, t_{p}, b_{1}, \ldots, b_{p}$, и аналогично трактуется равенство $q=0$ (для соответствующих наборов); миноры нулевого порядка, формально появляющиеся при этом, считаются равными единице.

ДокАЗАТЕЛЬСтво. Ввиду (4.1) и (4.4) минор в левой части (4.5) равен определителю следуюшего произведения прямоугольных матриц

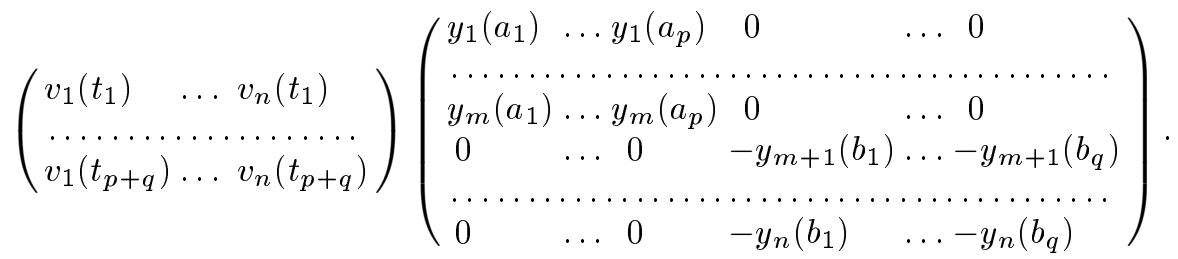

Отсюда по теореме Бине-Коши с учетом блочной структуры второго сомножителя следует справедливость (4.5), где суммирование ведется по наборам индексов, удовлетворяющим (4.7).

Аналогично, ввиду (4.1) и (4.4) минор в левой части (4.6) равен определителю следуюшего произведения матриц

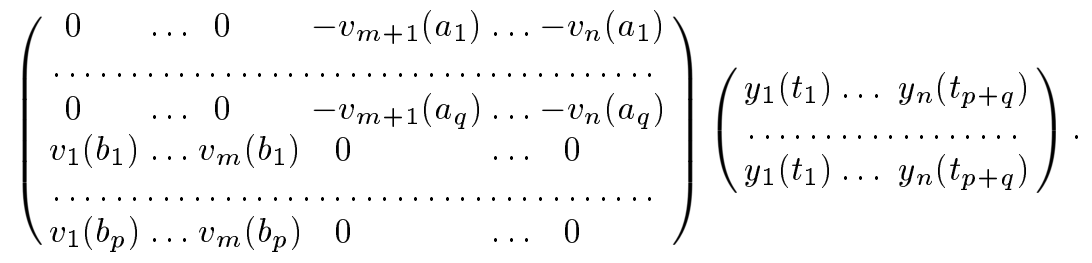

Отсюда по теореме Бине-Коши с учетом блочной структуры первого сомножителя следует справедливость (4.6). Лемма доказана. 
Лемма 4.2. Пусть $r=\max \{m, n-m\} u$

$$
a<a_{1}<\cdots<a_{r}<t_{1}<s_{1}<\cdots<t_{p}<s_{p}<b_{1}<\cdots<b_{r}<b .
$$

Тогда при $p=1,2, \ldots$ вьцполняются равенства

$$
\begin{aligned}
& G\left(\begin{array}{c}
t_{1}, \ldots, t_{p}, b_{1}, \ldots, b_{m} \\
a_{1}, \ldots, a_{m}, s_{1}, \ldots, s_{p}
\end{array}\right) \\
& \quad=G\left(\begin{array}{c}
b_{1}, \ldots, b_{m} \\
a_{1}, \ldots, a_{m}
\end{array}\right) x\left(t_{1}, s_{1}\right) \cdots x\left(t_{p}, s_{p}\right)(-1)^{p(m+1)}, \\
& G\left(\begin{array}{c}
a_{1}, \ldots, a_{n-m}, s_{1}, \ldots, s_{p} \\
t_{1}, \ldots, t_{p}, b_{1}, \ldots, b_{n-m}
\end{array}\right) \\
& \quad=G\left(\begin{array}{c}
a_{1}, \ldots, a_{n-m} \\
b_{1}, \ldots, b_{n-m}
\end{array}\right) x\left(s_{1}, t_{1}\right) \cdots x\left(s_{p}, t_{p}\right)(-1)^{p(n-m)},
\end{aligned}
$$

где $x(t, s)$ определяется формулой (4.3).

ДоКАЗАТЕЛЬСтво. Ввиду (4.1) и (4.8) минор, стоящий в левой части (4.9), равен следуюшему произведению определителей двух $(m+p) \times(m+p)$-матрищ блочной структуры

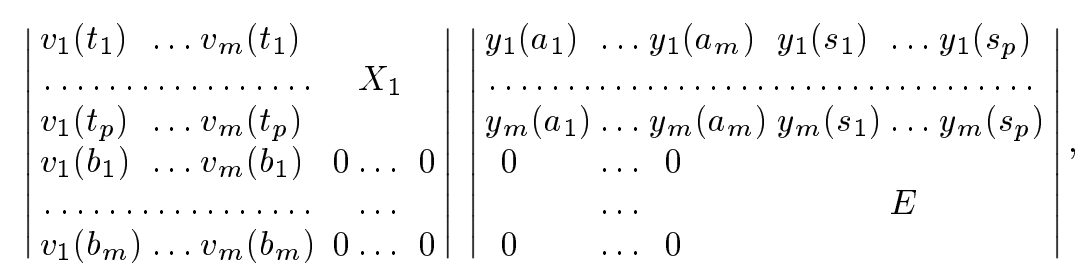

где блок $E$ представляет из себя единичную $(p \times p)$-матрицу, а $X_{1}-$ верхняя треугольная $(p \times p)$-матрица, имеющая вид

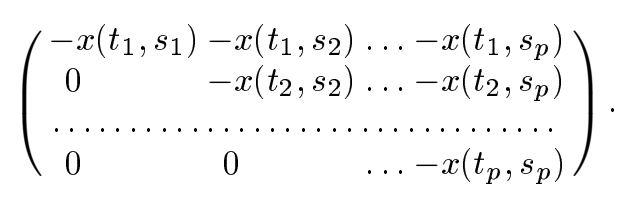

В свою очередь, ввиду специфики структуры матриц это произведение определителей равно

$$
(-1)^{p(m+1)} x\left(t_{1}, s_{1}\right) \cdots x\left(t_{p}, s_{p}\right) v\left(\begin{array}{c}
b_{1}, \ldots, b_{m} \\
1, \ldots, m
\end{array}\right) y\left(\begin{array}{c}
a_{1}, \ldots, a_{m} \\
1, \ldots, m
\end{array}\right)
$$

а значит, с учетом леммы 4.1 равно правой части (4.9).

Аналогично, ввиду (4.1) и (4.8) минор, стоящий в левой части (4.10), равен следующему произведению определителей двух $(n-m+p) \times(n-m+p)$-матриц 
блочной структуры

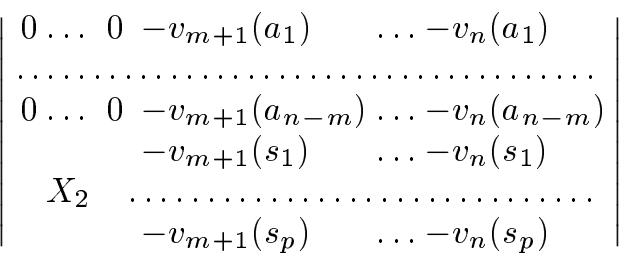

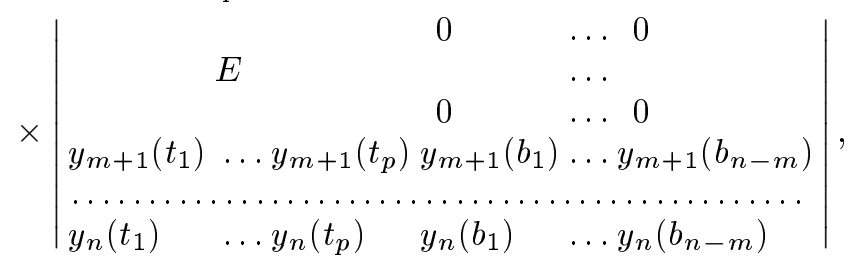

где блок $E$ - единичная $p \times p$-матрица, а $X_{2}$ - нижняя треугольная $(p \times p)$-матрица вида

$$
\left(\begin{array}{cccc}
x\left(s_{1}, t_{1}\right) & 0 & \ldots & 0 \\
x\left(s_{2}, t_{1}\right) & x\left(s_{2}, t_{2}\right) & \ldots & 0 \\
\ldots \ldots \ldots \ldots \ldots \ldots \ldots \ldots \ldots \ldots & \ldots \ldots \ldots \\
x\left(s_{p}, t_{1}\right) & x\left(s_{p}, t_{2}\right) & \ldots & x\left(s_{p}, t_{p}\right)
\end{array}\right) .
$$

Это произведение определителей ввиду структуры матриц и леммы 4.1 равно правой части (4.10), что означает справедливость (4.10). Лемма доказана.

Следующие две леммы выражают зависимость поведения системы функций $(4.2)$ в окрестности концов интервала $[a, b]$ от порядков $k_{1}, \ldots, k_{n}$ ведущих производных краевых условий (1.2). Для их формулировки и обоснования введем обозначения

$$
l_{i}=n-1-k_{i} \quad(i=\overline{1, n}) .
$$

Лемма 4.3. При положительных $h \rightarrow 0$ имеют место равенства

$$
\begin{array}{ll}
y_{i}(a+h)=h^{l_{i}} / l_{i} !+o\left(h^{l_{i}}\right) & (i=\overline{1, m}), \\
y_{i}(b-h)=(-1)^{i-m} h^{l_{i}} / l_{i} !+o\left(h^{l_{i}}\right) & (i=\overline{m+1, n}) .
\end{array}
$$

ДокАЗАТЕЛЬСТво. Для $i=\overline{1, m}$ из леммы 2.1 , примененной при $t=a$ к системе функций $\left\{v_{j}\right\}_{1}^{n} \backslash v_{i}$, и из леммы 2.2 следуют соответственно равенства

$$
\begin{gathered}
{\left[v_{1}, \ldots, v_{i-1}, v_{i+1}, \ldots, v_{n}\right](a+h)=\frac{1}{l_{i} !} v\left(\begin{array}{l}
\{0, \ldots, n-1\} \backslash k_{i} \\
\{1, \ldots, n\} \backslash i
\end{array}\right)(a) h^{l_{i}}+o\left(h^{l_{i}}\right),} \\
{\left[v_{1}, v_{2}, \ldots, v_{n}\right](a)=(-1)^{n+i} v\left(\begin{array}{l}
\{0, \ldots, n-1\} \backslash k_{i} \\
\{1, \ldots, n\} \backslash i
\end{array}\right)(a) .}
\end{gathered}
$$

Отсюда и из (4.2) следует справедливость (4.11).

Аналогично, при $i=\overline{m+1, n}$ из лемм $2.1,2.2$ следует, что

$$
\begin{gathered}
{\left[v_{1}, \ldots, v_{i-1}, v_{i+1}, \ldots, v_{n}\right](b-h)=\frac{(-1)^{l_{i}}}{l_{i} !} v\left(\begin{array}{l}
\{0, \ldots, n-1\} \backslash k_{i} \\
\{1, \ldots, n\} \backslash i
\end{array}\right)(b) h^{l_{i}}+o\left(h^{l_{i}}\right),} \\
{\left[v_{1}, v_{2}, \ldots, v_{n}\right](b)=(-1)^{k_{i}+m+1} v\left(\begin{array}{l}
\{0, \ldots, n-1\} \backslash k_{i} \\
\{1, \ldots, n\} \backslash i
\end{array}\right)(b) .}
\end{gathered}
$$


Отсюда и из (4.2) следует справедливость (4.12). Лемма доказана.

Попутно, не вдаваясь в подробности, отметим, что из доказанного утверждения следует, что порядки ведущих производных исходной задачи и порядки $k_{1}^{*}, \ldots, k_{n-m}^{*}$ и $k_{n-m+1}^{*}, \ldots, k_{n}^{*}$ ведущих производных нормированных краевых условий сопряженной задачи связаны соотношениями

$$
\begin{gathered}
\left\{l_{1}, \ldots, l_{m}\right\} \cup\left\{k_{1}^{*}, \ldots, k_{n-m}^{*}\right\}=\{0,1, \ldots, n-1\}, \\
\left\{l_{m+1}, \ldots, l_{n}\right\} \cup\left\{k_{n-m+1}^{*}, \ldots, k_{n}^{*}\right\}=\{0,1, \ldots, n-1\} .
\end{gathered}
$$

Лемма 4.4. При всех $\nu=\overline{1, m}$ и положительных $h \rightarrow 0$, если

$$
1 \leqslant i_{1}<\cdots<i_{\nu} \leqslant m
$$

mo

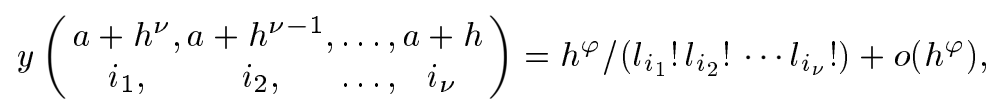

2дe

$$
\varphi=\nu l_{i_{1}}+(\nu-1) l_{i_{2}}+\cdots+l_{i_{\nu}} .
$$

Аналогично, при всех $\mu=\overline{1, n-m}$ и положительных $h \rightarrow 0$, если

$$
m+1 \leqslant j_{1}<\cdots<j_{\mu} \leqslant n
$$

mo

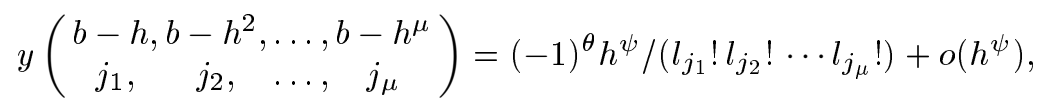

əde

$$
\psi=\mu l_{j_{1}}+(\mu-1) l_{j_{2}}+\cdots+l_{j_{\mu}}, \quad \theta=j_{1}+\cdots+j_{m}-m \mu-\frac{\mu(\mu-1)}{2} .
$$

ДоКАЗАТЕЛЬСТВО. При условии (4.13) из леммы 4.3 очевидным образом следует, что

$$
y\left(\begin{array}{c}
a+h^{\nu}, \ldots, a+h \\
i_{1}, \quad \ldots, i_{\nu}
\end{array}\right)=\frac{1}{l_{i_{1}} ! \cdots l_{i_{\nu}} !}\left|\begin{array}{c}
h^{\rho_{\nu 1}}+o\left(h^{\rho_{\nu 1}}\right) \ldots h^{\rho_{\nu \nu}}+o\left(h^{\rho_{\nu \nu}}\right) \\
\ldots \ldots \ldots \ldots \ldots \ldots \ldots \ldots \ldots \ldots \\
h^{\rho_{11}}+o\left(h^{\rho_{11}}\right) \ldots h^{\rho_{1 \nu}}+o\left(h^{\rho_{1 \nu}}\right)
\end{array}\right|,
$$

где $\rho_{p q}=p l_{i_{q}}(p=\overline{1, \nu} ; q=\overline{1, \nu})$.

Так как $k_{i_{1}}>\cdots>k_{i_{\nu}}$, то $l_{i_{1}}<\cdots<l_{i_{\nu}}$, а значит главный член разложения по степеням $h$ определителя в правой части (4.19) дает произведение элементов, стоящих на главной диагонали. Последнее означает справедливость представления (4.14), (4.15).

Аналогично, при условии (4.16) из леммы 4.3 следует, что

$$
y\left(\begin{array}{c}
b-h, \ldots, b-h^{\mu} \\
j_{1}, \ldots, \quad j_{\mu}
\end{array}\right)=\frac{(-1)^{j_{1}+\cdots+j_{\mu}-m \mu}}{l_{j_{1}} ! \cdots l_{j_{\mu}} !}\left|\begin{array}{c}
h^{\rho_{11}}+o\left(h^{\rho_{11}}\right) \cdots h^{\rho_{1 \mu}}+o\left(h^{\rho_{1 \mu}}\right) \\
\ldots \ldots \ldots \ldots \ldots \ldots \ldots \ldots \ldots \ldots \\
h^{\rho_{\mu 1}}+o\left(h^{\rho_{\mu 1}}\right) \cdots h^{\rho_{\mu \mu}}+o\left(h^{\rho_{\mu \mu}}\right)
\end{array}\right|,
$$

где $\rho_{p q}=p l_{j_{q}}(p=\overline{1, \mu} ; q=\overline{1, \mu})$.

Так как $k_{j_{1}}>\cdots>k_{j_{\mu}}$, то $l_{j_{1}}<\cdots<l_{j_{\mu}}$, а значит, главный член разложения по степеням $h$ определителя в правой части (4.20) дает произведение элементов, стоящих на побочной диагонали. Последнее, очевидно, означает справедливость (4.17), (4.18). Лемма доказана. 
ТЕОРема 4.1. Пусть при некотором $p \leqslant n$ и константе $\varepsilon_{p}$, по модулю равной единице, для миноров функции Грина выполнено условие

$$
\varepsilon_{p} G\left(\begin{array}{c}
t_{1}, t_{2}, \ldots, t_{p} \\
s_{1}, s_{2}, \ldots, s_{p}
\end{array}\right) \geqslant 0 \quad\left(a<\begin{array}{c}
t_{1}<t_{2}<\cdots<t_{p} \\
s_{1}<s_{2}<\cdots<s_{p}
\end{array}<\right) .
$$

Тогда при всех $q$ таких, что $0 \leqslant q \leqslant m, 0 \leqslant p-q \leqslant n-m$, справедливы соотношения

$$
\varepsilon_{p} v\left(\begin{array}{l}
t_{1}, t_{2}, \quad \ldots, \quad t_{p} \\
1, \ldots, q, m+1, \ldots, m+p-q
\end{array}\right) \geqslant 0 \quad\left(a<t_{1}<\cdots<t_{p}<b\right) .
$$

( $B$ (4.22) подпоследовательность номеров $1, \ldots, q(m+1, \ldots, m+p-q)$ при $q=0(p-q=0)$, естественно, считается пустой. $)$

ДоКАЗАТЕЛЬСТво. Из лемм 4.1 и 4.4 следует, что при всех $p \leqslant n-m, 0 \leqslant$ $p-q \leqslant n-m, a<t_{1}<\cdots<t_{p}<b$ и положительном $h \rightarrow 0$ минор

$$
G\left(\begin{array}{l}
t_{1}, \quad t_{2}, \\
a+h^{q}, a+h^{q-1}, \ldots, a+h, b-h, b-h^{2}, \ldots, b-h^{p-q}
\end{array}\right)
$$

равен

$$
\frac{(-1)^{\eta} h^{\zeta}}{l_{1} ! \cdots l_{q} ! l_{m+1} ! \cdots l_{m+p-q} !} v\left(\begin{array}{l}
t_{1}, t_{2}, \quad \ldots, \quad t_{p} \\
1, \ldots, q, m+1, \ldots, m+p-q
\end{array}\right)+o\left(h^{\zeta}\right),
$$

где

$$
\begin{gathered}
\zeta=\left(q l_{1}+(q-1) l_{2}+\cdots+l_{q}\right)+\left((p-q) l_{m+1}+(p-q-1) l_{m+2}+\cdots+l_{m+p-q}\right), \\
\eta=(p-q)+((m+1)+(m+2)+\cdots+(m+p-q)) \\
-m(p-q)-\frac{(p-q)(p-q-1)}{2}=2(p-q) .
\end{gathered}
$$

Следовательно, если у системы функций $v_{1}(t), \ldots, v_{n}(t)$ отличен от нуля некоторый минор

$$
v\left(\begin{array}{l}
t_{1}, t_{2}, \quad \ldots, \quad t_{p} \\
1, \ldots, q, m+1, \ldots, m+p-q
\end{array}\right),
$$

то при малых $h$ с ним совпадают по знаку все соответствующие миноры (4.23) функции Грина $G(t, s)$. Последнее с учетом (4.21) означает справедливость (4.22). Теорема доказана.

С учетом теорем 3.1 и 4.1 доказательство необходимости в теореме 1.1 становится практически очевиднњм и требует лишь перенумерации функций системы $\left\{v_{i}\right\}_{1}^{n}$ с соответствующей сменой знаков. Подробнее, пусть функция Грина $G(t, s)$ знакорегулярна, $\varepsilon_{1}, \ldots, \varepsilon_{n}$ - характеристики знаков соответствуюших миноров, $\varepsilon_{0}=1$. Покажем, что в $(a, b)$ справедливы соотношения

$$
\varepsilon_{k+l} v_{k l}(t)>0 \quad(k=\overline{0, m} ; l=\overline{0, n-m}) .
$$


Для проверки (4.24) положим при фиксированных $k$ и $l(k \leqslant m, l \leqslant n-m)$

$$
z_{i}(t)= \begin{cases}\varepsilon_{i-1} \varepsilon_{i} v_{i}(t) & \text { при } i=\overline{1, k} \text { и при } i=\overline{m+l+1, n}, \\ \varepsilon_{i-1} \varepsilon_{i} v_{m+i-k}(t) & \text { при } i=\overline{k+1, k+l}, \\ (-1)^{l} \varepsilon_{i-1} \varepsilon_{i} v_{i-l}(t) & \text { при } i=\overline{k+l+1, m+l} .\end{cases}
$$

Тогда ввиду теоремы 4.1 система функций $\left\{z_{i}(t)\right\}_{1}^{n}$ при $p=\overline{1, n-1}$ удовлетворяет условиям (3.1) теоремы 3.1 и ввиду фундаментальности системы $\left\{v_{i}\right\}_{1}^{n}$ удовлетворяет при $p=n-1$ условию (3.2). Поэтому из теоремы 3.1 следует, что $\left[z_{1}, z_{2}, \ldots, z_{k+l}\right](t)>0$ в $(a, b)$, а значит, справедливо (4.24), что завершает доказательство необходимости в теореме 1.1 .

Отметим, что в приведенных рассуждениях вообще не использовалось знакопостоянство миноров функции Грина, порядок которых больше $n$, а знакопостоянство миноров $n$-го порядка по сушеству было использовано только для уточнения знака $\varepsilon_{n}$, поскольку отличие от нуля вронскиана $v_{m n-m}$ заведомо было обеспечено самим сушествованием функции Грина. Поэтому все наши рассуждения справедливы и в случае, если заранее известна не знакорегулярность функции Грина, а лишш справедливость условия (А) из $§ 1$. Таким образом, уже выполнение условия (A) обеспечивает соотношения (4.24), если только при этом через $\varepsilon_{n}$ обозначить характеристику знака вронскиана $v_{m n-m}$. Сказанное также означает, что, когда будет доказана достаточность в теореме 1.1, то автоматически будет доказана и достаточность условия (А) для знакорегулярности функции Грина.

Доказав (4.24), мы частично доказали и вторую часть теоремы 1.1. Для полного обоснования этой части осталось еше показать, что в знакорегулярном случае при условии $k>\min (m, n-m)$ справедливо равенство

$$
\varepsilon_{k} \varepsilon_{k-1}=(-1)^{n-m} .
$$

В случае $\min (m, n-m)<k \leqslant n$ это равенство непосредственно вытекает из (4.24) и следствия 2.1. Для доказательства (4.25) при остальных $k$ воспользуемся леммой 4.2. Отметим, что миноры, фигурируюшие в (4.9), (4.10), у знакорегулярной функции Грина при условии (4.8) обязательно отличны от нуля [3, теорема 5]. Поэтому из справедливости $(4.25)$ при $\min (m, n-m)<k \leqslant n$ и леммы 4.2 следует, что при $a<t<s<b$ вьполнены неравенства

$$
x(s, t)>0, \quad(-1)^{n-1} x(t, s)>0,
$$

а значит, ввиду (4.10) равенство (4.25) выполнено и при $k>n$.

Из (4.24) и (4.25) следует, что если каждую функцию в системе $\left\{v_{i}\right\}_{1}^{n}$ умножить на $(-1)^{n-m}$, то вронскианы вида (1.9) вновь полученной системы функций тоже будут отличными от нуля в $(a, b)$, будут совпадать по знаку при совпадении порядков и, дополнительно, будут совпадать по знаку, когда их порядок $\geqslant \min (m, n-m)$. Это означает, что в случае знакорегулярности функции Грина обязательно выполняется условие (Б) §1. Кроме того, из (4.24) и (4.25) следует, что функция Грина может быть осцилляпионным ядром лишь с точностью до множителя $(-1)^{n-m}$ и в случае осцилляционности ядра $(-1)^{n-m} G(t, s)$ выполняются условия

$$
\varepsilon_{i}=(-1)^{(n-m) i} \quad(i=0,1, \ldots) .
$$

Сказанное, в частности, означает справедливость необходимости в теоремах 1.2 и 1.3, поскольку, очевидно, фигурируюшие в них системы функций с учетом (4.26), (4.24) можно получить домножением на $(-1)^{n-m}$ всех функций системы $\left\{v_{i}\right\}_{1}^{n}$. 


\section{§5. Знакорегулярность и свойство оператора $L$ не понижать числа перемен знака}

Начиная с данного параграфа, переходим к обоснованию достаточности в теоремах 1.1-1.3. Как уже отмечалось, основньм инструментом при этом будет доказанный в [3] критерий знакорегулярности функции Грина, выражающий эквивалентность знакорегулярности свойству оператора $L$ не понижать числа перемен знака.

Далее (следуя, в основном, [4], [14], [35]) используются два варианта подсчета числа перемен знака вещественного вектора $\xi=\left(\xi_{0}, \xi_{1}, \ldots, \xi_{n}\right)$, дающие соответственно две характеристики - минимальное число перемен знака $S^{-}(\xi)=$ $S^{-}\left(\xi_{0}, \xi_{1}, \ldots, \xi_{n}\right)$ и максимальное число перемен знака $S^{+}(\xi)=S^{+}\left(\xi_{0}, \xi_{1}, \ldots, \xi_{n}\right)$. Для вектора, не содержашего нулевых координат, эти характеристики совпадают и полагаются равными числу индексов $i(i=\overline{1, n})$, при которых $\xi_{i-1} \xi_{i}<0$. Для ненулевого вектора, у которого есть нулевые координаты, число $S^{-}(\xi)$ полагается равным аналогичной характеристике вектора, получающегося из $\xi$ вычеркиванием всех нулевых координат, а $S^{+}(\xi)$ полагается равным максимально возможному значению аналогичной характеристики у векторов, получающихся из исходного заменой нулевых координат на \pm 1 . Для нулевого $(n+1)$-мерного вектора $\xi$ по определению считается $S^{-}(\xi)=-1$ и $S^{+}(\xi)=n+1$.

Следуя [3], под числом перемен знака $S(f)$ измеримой на $[a, b]$ функции $f(t)$ в данной работе понимается аналог величины $S^{-}(\xi)$ для вектора. Если $f(t)$ эквивалентна нулю, то по определению $S(f)=-1$. В противном случае положим $S(f)=p<\infty$, если сушествуют точки $t_{i}\left(a=t_{0}<t_{1}<\cdots<t_{p+1}=b\right)$ такие, что на каждом промежутке $\left[t_{k}, t_{k+1}\right], k=\overline{0, p}$, функция $f(t)$ не эквивалентна нулю и для некоторого $c \neq 0 c(-1)^{k} f(t) \geqslant 0$ почти всюду на $\left[t_{k}, t_{k+1}\right]$. В случае отсутствия таких конечных наборов $\left\{t_{i}\right\}$, полагаем $S(f)=\infty$. Если $f$ является непрерывной функцией, то, очевидно, $S(f)=\sup S^{-}\left(f\left(t_{0}\right), f\left(t_{1}\right), \ldots, f\left(t_{p}\right)\right)$ по всем возрастающим наборам $(a \leqslant) t_{0}<\cdots<t_{k}(\leqslant b), k=0,1, \ldots$.

Если для $d \in[a, b]$ сушествуют $\Delta>0$ и $c_{1} \neq 0\left(c_{2} \neq 0\right)$ такие, что $d+\Delta \in[a, b]$ $(d-\Delta \in[a, b])$ и на $[d, d+\Delta]([d-\Delta, d])$ функция $f(t)$ не эквивалентна нулю и почти всюду $c_{1} f(t) \geqslant 0\left(c_{2} f(t) \geqslant 0\right)$, то положим $\operatorname{sign}_{1} f(d+)=\operatorname{sign} c_{1}$ $\left(\operatorname{sign}_{1} f(d-)=\operatorname{sign} c_{2}\right)$. Эти величины характеризуют знаки первых (за исключением множества меры нуль) ненулевых значений справа и слева от точки $d$. Очевидно, что если $0 \leqslant S(f)=p<\infty$, то характеристики $\operatorname{sign}_{1} f(a+), \operatorname{sign}_{1} f(b-)$ заведомо определены и $\operatorname{sign}_{1} f(b-)=(-1)^{p} \operatorname{sign}_{1} f(a+)$.

Для абсолютно непрерывной на $[a, b]$ функции $f(t)$ легко проверяются следующие свойства, очевидным образом связанные по смыслу с теоремой Ролля.

$1^{\circ}$. Если $f(a)=f(b)=0$ и $S(f)=0$, то $S\left(f^{\prime}\right) \geqslant 1$.

$2^{\circ}$. Если $S(f)=\infty$, то и $S\left(f^{\prime}\right)=\infty$.

$3^{\circ}$. Если $a \leqslant d<b, f(d)=0$ и определены $\operatorname{sign}_{1} f(d+), \operatorname{sign}_{1} f^{\prime}(d+)$, то $\operatorname{sign}_{1} f^{\prime}(d+)=\operatorname{sign}_{1} f(d+)$. Если $a<d \leqslant b, f(d)=0$ и определены $\operatorname{sign}_{1} f(d-)$, $\operatorname{sign}_{1} f^{\prime}(d-)$, то $\operatorname{sign}_{1} f^{\prime}(d-)=-\operatorname{sign}_{1} f(d-)$.

$4^{\circ}$. Если $0 \leqslant S\left(f^{\prime}\right)<\infty$, то $S\left(f^{\prime}\right) \geqslant S(f)-S^{-}\left(\xi_{0}^{a}, \xi_{1}^{a}\right)+S^{-}\left(\xi_{0}^{b}, \xi_{1}^{b}\right)$, где $\xi_{0}^{a}=\operatorname{sign}_{1} f(a+), \xi_{1}^{a}=\operatorname{sign}_{1} f^{\prime}(a+), \xi_{0}^{b}=\operatorname{sign}_{1} f(b-), \xi_{1}^{b}=\operatorname{sign}_{1} f^{\prime}(b-)$.

Далее через $\mathscr{D}$ обозначается множество функций, имеющих абсолютно непрерывную на $[a, b]$ производную $(n-1)$-го порядка и удовлетворяющих краевым условиям (1.2), а подмножество таких функций, дополнительно удовлетворяющих условию $0 \leqslant S(L x)<\infty$, обозначается через $\mathscr{D}_{1}$. 
TeOpema 5.1 [3].

$1^{\circ}$. Для того чтобъ задача (1.1)-(1.2) обладала знакорегулярной (а значит и сильно знакорегулярной) функиией Грина, необходимо и достаточно, чтобъ оператор $L$ не понижал числа перемен знака на $\mathscr{D}$, т.е. выполнялось условие

$$
S(L x) \geqslant S(x) \quad(x \in \mathscr{D}) .
$$

$2^{\circ}$. Если функиия Грина задачи существует и знакорегулярна, и для некоторого $x \in \mathscr{D}_{1}$ имеет место равенство

$$
S(L x)=S(x)=p,
$$

mo

$$
\operatorname{sign}_{1} x(a+) \operatorname{sign}_{1} L x(a+)=\varepsilon_{p} \varepsilon_{p+1}
$$

где $\varepsilon_{1}, \varepsilon_{2}, \ldots$ - характеристики знаков миноров функции Грина, а $\varepsilon_{0}=1$.

ЗАмЕчАнИЕ. Очевидно, что условие (5.1) эквивалентно условию

$$
S(L x) \geqslant S(x) \quad\left(x \in \mathscr{D}_{1}\right)
$$

с одновременным требованием неособенности задачи (1.1)-(1.2). Поэтому, если заведомо известно о существовании функции Грина, то для доказательства ее знакорегулярности достаточно проверить условие (5.4).

Удобство приведенного критерия для доказательства достаточных признаков осцилляционности и знакорегулярности в значительной мере связано с тем обстоятельством, что в случае знакорегулярности, как следует из уже доказанной необходимости в теореме 1.1, дифференциальный оператор представим в виде

$$
L x \equiv r_{0} \frac{d}{d t} r_{1} \frac{d}{d t} \cdots r_{n-1} \frac{d}{d t} r_{n} x,
$$

где $r_{0}, r_{1}, \ldots, r_{i}$ - достаточно гладкие положительные в $(a, b)$ функции. Если заранее известно, что оператор представим в виде (5.5), то, например, для краевых условий (1.7), в случае существования функции Грина $G(t, s)$, из теоремы 5.1 и соображений типа теоремы Ролля автоматически следует осцилляционность ядра $(-1)^{n-m} G(t, s)$. Далее будем использовать теорему 5.1 с некоторьми модификациями теоремы Ролля, с целью формулировки которых введем обозначения для соответствуюших разложению (5.5) квазипроизводных

$$
D_{0} x=x, \quad D_{i} x=\frac{d}{d t}\left(r_{n-i+1} D_{i-1} x\right) \quad(i=\overline{1, n}) .
$$

Лемма 5.1. Пусть оператор L имеет на интервале $(a, b)$ представление (5.5) и $x \in \mathscr{D}_{1}$. Тогда для соответствующих квазипроизводных (5.6) при $i=\overline{0, n}$ определень характеристики

$$
\xi_{i}^{a}=\operatorname{sign}_{1} D_{i} x(a+), \quad \xi_{i}^{b}=\operatorname{sign}_{1} D_{i} x(b-),
$$

и для векторов

$$
\xi^{a}=\left(\xi_{0}^{a}, \xi_{1}^{a}, \ldots, \xi_{n}^{a}\right), \quad \xi^{b}=\left(\xi_{0}^{b}, \xi_{1}^{b}, \ldots, \xi_{n}^{b}\right)
$$

выполнено соотночение

$$
S(L x)=S\left(D_{n} x\right) \geqslant S(x)-S^{-}\left(\xi^{a}\right)+S^{-}\left(\xi^{b}\right) .
$$


ДокаЗАТЕЛЬство практически очевидно. Из $x \in \mathscr{D}_{1}$ и теоремы Ролля следует, что $0 \leqslant S\left(D_{i} x\right)<\infty$ при всех $i=\overline{0, n}$. Отсюда и определения характеристик $\operatorname{sign}_{1}$ следует существование (5.7). Справедливость (5.9) вытекает из следующего очевидного обобщения теоремы Ролля

$$
S\left(D_{i+1} x\right) \geqslant S\left(D_{i} x\right)-S^{-}\left(\xi_{i+1}^{a}, \xi_{i}^{a}\right)+S^{-}\left(\xi_{i+1}^{b}, \xi_{i}^{b}\right)
$$

и того простого факта, что если все $\xi_{i} \neq 0(i=\overline{0, n})$, то

$$
S^{-}\left(\xi_{0}, \xi_{1}, \ldots, \xi_{i+1}\right)=S^{-}\left(\xi_{0}, \xi_{1}, \ldots, \xi_{i}\right)+S^{-}\left(\xi_{i}, \xi_{i+1}\right) \quad(i=\overline{1, n-1}) .
$$

Лемма доказана.

Из теоремы 5.1 и леммы 5.1 видно, что если для неособой краевой задачи удастся установить, что $L$ представим в мультипликативном виде, а краевые условия таковы, что для каждого $x \in \mathscr{D}_{1}$ при некотором разложении (5.5) векторы (5.8) удовлетворяют неравенству $S^{-}\left(\xi^{b}\right) \geqslant S^{-}\left(\xi^{a}\right)$, то функция Грина краевой задачи знакорегулярна. Далее это соображение будет использоваться для доказательства достаточности в теоремах 1.1-1.3, а в качестве более простого примера его использования здесь докажем следующую теорему Калаффати-Гантмахера-Крейна-Карлина (ср. [20], [22]).

ТЕОрема 5.2. Пусть оператор $L$ задан на интервале $[a, b]$ в виде (5.5), а краевые условия имеют вид

$$
\begin{aligned}
& \sum_{k=1}^{n} \alpha_{i k}\left(D_{k-1} x\right)(a)=0 \quad(i=\overline{1, m}) \\
& \sum_{k=1}^{n} \beta_{j k}\left(D_{k-1} x\right)(b)=0 \quad(j=\overline{1, n-m}) .
\end{aligned}
$$

Пусть все отличнье от нуля миноры т-го порядка матрицьь

$$
A=\left\|(-1)^{k} \alpha_{i k}\right\| \quad(i=\overline{1, m}, k=\overline{1, n})
$$

имеют одинаковые знаки и то же справедливо для миноров $(n-m)$-го порядка матриць

$$
B=\left\|\beta_{j k}\right\| \quad(j=\overline{1, n-m}, k=\overline{1, n}) .
$$

Тогда если краевая задача является неособой и $G(t, s)$ - ее функиия Грина, то $(-1)^{n-m} G(t, s)$ является осиилляиионным ядром.

ДоказАтельство. Рассмотрим наряду с $(n+1)$-мерными векторами $\xi^{a}$ и $\xi^{b}$, определяемыми условиями $(5.7),(5.8),(n+1)$-мерный вектор $\widetilde{\xi}^{a}=\left(\widetilde{\xi}_{0}^{a}, \widetilde{\xi}_{1}^{a}, \ldots, \widetilde{\xi}_{n}^{a}\right)$, где $\widetilde{\xi}_{i}^{a}=(-1)^{i} \xi_{i}^{a}(i=\overline{0, n})$, и два $n$-мерных вектора $\widetilde{\eta}^{a}=\left(\widetilde{\eta}_{0}^{a}, \widetilde{\eta}_{1}^{a}, \ldots, \widetilde{\eta}_{n-1}^{a}\right)$ и $\eta^{b}=\left(\eta_{0}^{b}, \eta_{1}^{b}, \ldots, \eta_{n-1}^{b}\right)$, где $\widetilde{\eta}_{j}^{a}=(-1)^{j} D_{j} x(a)$ и $\eta_{j}^{b}=D_{j} x(b)(j=\overline{0, n-1})$.

Легко видеть, что если при некотором $j<n$ вьполнено равенство $\widetilde{\eta}_{j}^{a}=0$, то $\widetilde{\xi}_{j}^{a}=-\widetilde{\xi}_{j+1}^{a}(\neq 0)$, а если $\eta_{j}^{b}=0$, то $\xi_{j}^{b}=-\xi_{j+1}^{b}(\neq 0)$, и поэтому

$$
S^{+}\left(\widetilde{\eta}^{a}\right) \leqslant S^{-}\left(\widetilde{\xi}^{a}\right), \quad S^{+}\left(\eta^{b}\right) \leqslant S^{-}\left(\xi^{b}\right) .
$$

Известно [4], [35], что у $(m \times n)$-матрицы $A$ ранга $m$ все ненулевые миноры порядка $m$ имеют одинаковые знаки в том и только том случае, когда из того, что 
$S^{+}(\xi)<m\left(\xi \in \mathbb{R}^{n}\right)$, обязательно следует, что $0 \leqslant S^{-}(A \xi)<m$. Поэтому с учетом (5.10) и условий теоремы, наложенных на матрицы $A$ и $B$,

$$
S^{+}\left(\widetilde{\eta}^{a}\right) \geqslant m, \quad S^{+}\left(\eta^{b}\right) \geqslant n-m .
$$

Из (5.11), (5.12) и очевидного равенства $S^{-}\left(\xi^{a}\right)+S^{-}\left(\widetilde{\xi}^{a}\right)=n$ следует, что $S^{-}\left(\widetilde{\xi}^{a}\right) \geqslant m, S^{-}\left(\xi^{b}\right) \geqslant n-m, S^{-}\left(\xi^{a}\right) \leqslant n-m$. Следовательно, $S^{-}\left(\xi^{b}\right) \geqslant S^{-}\left(\xi^{a}\right)$ и равенство возможно лишь при $S^{-}\left(\xi^{b}\right)=S^{-}\left(\xi^{a}\right)=n-m$. В свою очередь, по лемме 5.1 это означает, что выполнено условие (5.1) и в случае $S(L x)=S(x)$ обязательно $S^{-}\left(\xi^{b}\right)=S^{-}\left(\xi^{a}\right)=n-m$, а значит,

$$
\operatorname{sign}_{1} x(a+) \operatorname{sign}_{1} L x(a+)=(-1)^{n-m} .
$$

По теореме 5.1 это значит, что ядро $(-1)^{n-m} G(t, s)$ является осцилляционным, что завершает доказательство теоремы 5.2 .

\section{§6. Общая схема доказательства достаточности в теоремах 1.1-1.3. Завершение доказательства теоремы 1.3}

В данном параграфе будет уточнена общая схема доказательства достаточности для теорем 1.1-1.3, а затем будет полностью завершено доказательство наиболее простой из них - теоремы 1.3.

На протяжении всего параграфа, если не оговариваются дополнительные условия, считается, что $\left\{v_{i}\right\}_{1}^{n}$ является фундаментальной системой уравнения (1.6), удовлетворяюшей условиям (1.11). Во многих рассуждениях этого и следуюшего параграфов приходится оперировать довольно громоздкими вронскианньми выражениями, в связи с чем наряду с обозначениями (1.9) для вронскианов из функций системы $\left\{v_{i}\right\}_{1}^{n}$ введем еще несколько сокращенных обозначений. Характеристики знаков вронскианов $v_{i j}$ далее обозначаются через

$$
\sigma_{i j}=\sigma_{i j}(t)=\operatorname{sign} v_{i j}(t) \quad(i=\overline{0, m} ; j=\overline{0, n-m}) .
$$

Если $x(t) \in \mathscr{D}$, то обозначим

$x_{i j}(t)=\sigma_{i j}(t)\left[v_{1}, v_{2}, \ldots, v_{i}, v_{m+1}, v_{m+2}, \ldots, v_{m+j}, x\right](t) \quad(i=\overline{0, m} ; j=\overline{0, n-m})$,

где нулевые индексы в $x_{0 j}$ и $x_{i 0}$ означают, что речь идет о вронскианах, не зависящих соответственно от $v_{i}(i \leqslant m)$ и от $v_{m+j}(1 \leqslant j)$, и по определению $x_{00}(t)=x(t)$. Кроме того, если $x(t) \in \mathscr{D}_{1}$, то обозначим

$$
\xi_{i j}^{a}=\operatorname{sign}_{1} x_{i j}(a+), \quad \xi_{i j}^{b}=\operatorname{sign}_{1} x_{i j}(b-)
$$

(сушествование соответствуюших характеристик будет установлено при доказательстве леммы 6.2).

В ходе доказательства достаточности заметную роль будет играть следующее известное вронскианное тождество (см. [38]), фактически являюшееся вронскианњім аналогом уже использованного в $\S 3$ детерминантного тождества Сильвестра.

Лемма 6.1. Пусть $z_{1}(t), z_{2}(t), \ldots, z_{k+2}(t)$ достаточно гладкие функиии такие, что $\left[z_{1}, z_{2}, \ldots, z_{k+1}\right](t) \neq 0$. Тогда

$$
\frac{d}{d t}\left(\frac{\left[z_{1}, \ldots, z_{k}, z_{k+2}\right](t)}{\left[z_{1}, \ldots, z_{k}, z_{k+1}\right](t)}\right)=\frac{\left[z_{1}, \ldots, z_{k}\right](t)\left[z_{1}, \ldots, z_{k}, z_{k+1}, z_{k+2}\right](t)}{\left[z_{1}, \ldots, z_{k}, z_{k+1}\right]^{2}(t)}
$$


Если в (6.1) выполнить дифференцирование дроби в левой части и умножить обе части на $\left[z_{1}, \ldots, z_{k}, z_{k+1}\right]^{2}(t)$, то получится обычное детерминантное тождество Сильвестра для матрицы $\left\|z_{i}^{(j-1)}(t)\right\|_{1}^{k+2}$.

Из леммы 6.1 следует, что если $v_{i+1 j}(t) \neq 0$, то

$$
\frac{d}{d t}\left(\frac{x_{i j}(t)}{\left|v_{i+1 j}(t)\right|}\right)=\frac{\left|v_{i j}(t)\right|}{v_{i+1 j}^{2}(t)} x_{i+1 j}(t)
$$

и, аналогично, если $v_{i j+1}(t) \neq 0$, то

$$
\frac{d}{d t}\left(\frac{x_{i j}(t)}{\left|v_{i j+1}(t)\right|}\right)=\frac{\left|v_{i j}(t)\right|}{v_{i j+1}^{2}(t)} x_{i j+1}(t) .
$$

Лемма 6.2. Пусть $x(t) \in \mathscr{D}_{1}$ и для некоторого набора пар индексов

$$
\left(i_{0}, j_{0}\right),\left(i_{1}, j_{1}\right), \ldots,\left(i_{n}, j_{n}\right)
$$

такого, что $i_{k}+j_{k}=k \quad(k=\overline{0, n}) u$

$$
0=i_{0} \leqslant i_{1} \leqslant \cdots \leqslant i_{n}=m, 0=j_{0} \leqslant j_{1} \leqslant \cdots \leqslant j_{n}=n-m,
$$

вронскианы $v_{i_{k} j_{k}}(t)(k=\overline{0, n})$ в $(a, b)$ не обращаются в нуль. Тогда

$$
S(L x) \geqslant S(x)-S^{-}\left(\xi_{i_{0} j_{0}}^{a}, \xi_{i_{1} j_{1}}^{a}, \ldots, \xi_{i_{n} j_{n}}^{a}\right)+S^{-}\left(\xi_{i_{0} j_{0}}^{b}, \xi_{i_{1} j_{1}}^{b}, \ldots, \xi_{i_{n} j_{n}}^{b}\right) .
$$

ДоКАЗАТЕЛЬСТво. Из условий леммы и (6.2), (6.3) следует, что для оператора $L$ в $(a, b)$ имеет место представление (5.5), (5.6), где

$$
\begin{gathered}
r_{n}=\frac{1}{\left|v_{i_{1} j_{1}}\right|}, \quad r_{n-k}=\frac{\left|v_{i_{k} j_{k}}\right|^{2}}{\left|v_{i_{k-1} j_{k-1}}\right|\left|v_{i_{k+1} j_{k+1}}\right|} \quad(k=\overline{1, n-1}), \quad r_{0}=\frac{\left|v_{i_{n} j_{n}}\right|}{\left|v_{i_{n-1} j_{n-1}}\right|}, \\
D_{0} x=x_{i_{0} j_{0}}=x, \quad D_{k} x=\frac{d}{d t}\left(r_{n-k+1} D_{k-1} x\right)=\frac{\mid v_{i_{k-1} j_{k-1} \mid} x_{i_{k} j_{k}}}{\left|v_{i_{k} j_{k}}\right|^{2}} \quad(k=\overline{1, n}) .
\end{gathered}
$$

Отсюда и из леммы 5.1 следует, что при $k=\overline{0, n}$ существуют характеристики

$$
\begin{aligned}
& \xi_{i_{k} j_{k}}^{a}=\operatorname{sign}_{1} x_{i_{k} j_{k}}(a+)=\operatorname{sign}_{1} D_{k} x(a+), \\
& \xi_{i_{k} j_{k}}^{b}=\operatorname{sign}_{1} x_{i_{k} j_{k}}(b-)=\operatorname{sign}_{1} D_{k} x(b-)
\end{aligned}
$$

и выполнено (6.5). Лемма доказана.

Общая схема доказательства достаточности в теоремах 1.1-1.3 ввиду теоремы 5.1 и леммы 6.2 заключается в доказательстве существования для каждого $x(t) \in \mathscr{D}_{1}$ набора пар (6.4), удовлетворяюшего условиям леммы 6.2 , при котором

$$
S^{-}\left(\xi_{i_{0} j_{0}}^{a}, \xi_{i_{1} j_{1}}^{a}, \ldots, \xi_{i_{n} j_{n}}^{a}\right) \leqslant S^{-}\left(\xi_{i_{0} j_{0}}^{b}, \xi_{i_{1} j_{1}}^{b}, \ldots, \xi_{i_{n} j_{n}}^{b}\right) .
$$

Наиболее просто это делается для теоремы 1.3, а две другие теоремы, особенно теорема 1.1 , требуют сушественно более тонких рассуждений. Тем не менее во всех трех случаях важньм моментом является вывод из следующих двух лемм. 
Лемма 6.3. Пусть $x(t) \in \mathscr{D}_{1}$ и для набора пар индексов (6.4) выполнены условия лемми 6.2. Тогда, если при некотором $k<n$

$$
\lim _{t \rightarrow a+0} x_{i_{k} j_{k}}(t) / v_{i_{k+1} j_{k+1}}(t)=0,
$$

то $\xi_{i_{k} j_{k}}^{a}=\xi_{i_{k+1} j_{k+1}}^{a}$, и, аналогично, если при некотором $k<n$

$$
\lim _{t \rightarrow b-0} x_{i_{k} j_{k}}(t) / v_{i_{k+1} j_{k+1}}(t)=0,
$$

$m o \xi_{i_{k} j_{k}}^{b}=-\xi_{i_{k+1}}^{b} j_{k+1}$.

Справедливость этого утверждения очевидным образом вытекает из (6.2), (6.3) и определения характеристик $\xi_{i j}^{a}, \xi_{i j}^{b}$.

Лемма 6.4. Пусть $x(t) \in \mathscr{D}$. Тогда

$$
\begin{aligned}
\lim _{t \rightarrow a+0} x_{i n-m}(t) / v_{i+1 n-m}(t)=0 & (i=\overline{0, m-1}), \\
\lim _{t \rightarrow b-0} x_{m j}(t) / v_{m j+1}(t)=0 & (j=\overline{0, n-m-1}) .
\end{aligned}
$$

ДокАЗАТЕЛЬСТво. Для обоснования (6.7) рассмотрим две матрицы

$$
\begin{aligned}
& \left(\begin{array}{llllll}
v_{1}(a) & \ldots & v_{i+1}(a) & v_{m+1}(a) & \ldots & v_{n}(a) \\
\ldots \ldots \ldots \ldots & \ldots & \ldots & \ldots & \ldots & \ldots \ldots \ldots \ldots \\
v_{1}^{(n-1)}(a) & \ldots & v_{i+1}^{(n-1)}(a) & v_{m+1}^{(n-1)}(a) & \ldots & v_{n}^{(n-1)}(a)
\end{array}\right),
\end{aligned}
$$

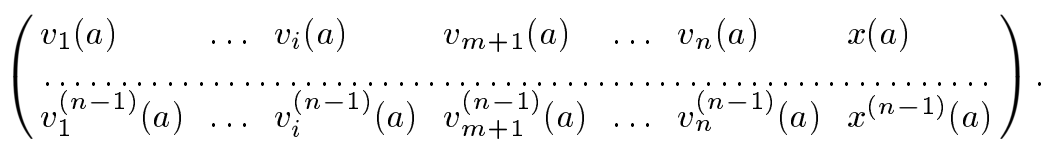

Очевидно, что ранг первой из них равен $i+1+n-m$, а ранг второй равен $i+n-m$, причем из линейной независимости некоторого набора строк второй матрицы следует линейная независимость строк с теми же номерами первой матрицы. Поэтому из леммы 2.1 следует справедливость (6.7). Точно так же обосновывается и равенство (6.8). Лемма доказана.

Из лемм 6.3 и 6.4 очевидным образом вытекает

СлеДСтвиЕ 6.1. Если $x(t) \in \mathscr{D}_{1}, m o$

$$
\begin{aligned}
S^{-}\left(\xi_{0 n-m}^{a}, \xi_{1 n-m}^{a}, \ldots, \xi_{m n-m}^{a}\right) & =0 \\
S^{-}\left(\xi_{m 0}^{b}, \xi_{m 1}^{b}, \ldots, \xi_{m n-m}^{b}\right) & =n-m
\end{aligned}
$$

$u$

$$
\begin{aligned}
& S^{-}\left(\xi_{00}^{a}, \xi_{01}^{a}, \ldots, \xi_{0 n-m}^{a}, \xi_{1 n-m}^{a}, \ldots, \xi_{m n-m}^{a}\right) \leqslant n-m, \\
& S^{-}\left(\xi_{00}^{b}, \xi_{10}^{b}, \ldots, \xi_{m 0}^{b}, \xi_{m 1}^{b}, \ldots, \xi_{m n-m}^{b}\right) \geqslant n-m .
\end{aligned}
$$

Следует обратить внимание, что в неравенствах (6.10), (6.11) фигурируют два разных набора пар индексов, а для получения неравенства вида (6.6) достаточно было бы указать аналогичную пару неравенств при одинаковых наборах индексов. В условиях теоремы 1.3 это удается сделать с помощью следующей леммы. 
Лемма 6.5. Пусть $x(t) \in \mathscr{D}$. Тогда, если в условиях $(1.2) k_{1}=m-1$, то

$$
\lim _{t \rightarrow a+0} x_{i 0}(t) / v_{i+10}(t)=0 \quad(i=\overline{0, m-1}),
$$

u, аналогично, если в условиях (1.2) $k_{m+1}=n-m-1$, то

$$
\lim _{t \rightarrow b-0} x_{0 j}(t) / v_{0 j+1}(t)=0 \quad(j=\overline{0, n-m-1}) .
$$

ДоКАЗАТЕЛЬСТво. Ограничимся доказательством справедливости (6.12) при $k_{1}=m-1$, поскольку доказательство (6.13) при $k_{m+1}=n-m-1$ вполне аналогично.

При $k_{1}=m-1$ ввиду (1.11) и (1.2) выполнено неравенство $\left[v_{1}, \ldots, v_{m}\right](a) \neq 0$. Поэтому при $i=\overline{0, m-1}$ и $x \in \mathscr{D}$ матрицы

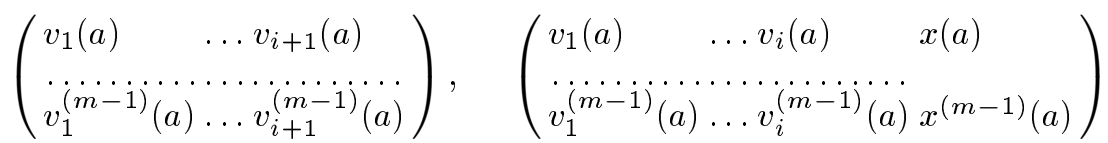

имеют соответственно ранги $i+1$ и $i$. Это обстоятельство ввиду леммы 2.1 влечет справедливость (6.12). Лемма доказана.

Из лемм $6.5,6.3$ вытекает, что для $x \in \mathscr{D}_{1}$ при $k_{1}=m-1$ выполнено неравенство

$$
S^{-}\left(\xi_{00}^{a}, \xi_{10}^{a}, \ldots, \xi_{m 0}^{a}, \xi_{m 1}^{a}, \ldots, \xi_{m n-m}^{a}\right) \leqslant n-m
$$

и, аналогично, при $k_{m+1}=n-m-1$ вьполнено неравенство

$$
S^{-}\left(\xi_{00}^{b}, \xi_{01}^{b}, \ldots, \xi_{0 n-m}^{b}, \xi_{1 n-m}^{b}, \ldots, \xi_{m n-m}^{b}\right) \geqslant n-m
$$

Теперь можно доказать достаточность в теореме 1.3.

Итак, пусть система функций $\left\{v_{i}(t)\right\}_{1}^{n}$ является фундаментальной системой решений уравнения (1.6), удовлетворяющей условию (1.11), и, кроме того, либо $k_{1}=m-1$ и все вронскианы $v_{0 j}(j=\overline{1, n-m}), v_{i n-m}(i=\overline{1, m})$ положительны в $(a, b)$, либо $k_{m+1}=n-m-1$ и в $(a, b)$ положительны все вронскианы $v_{i 0}$ $(i=\overline{1, m}), v_{m j}(j=\overline{1, n-m})$. Тогда из леммы 6.2 , за счет $(6.14),(6.11)$, если $k_{1}=m-1$, и за счет $(6.15),(6.10)$, если $k_{m+1}=n-m-1$, следует выполнение условия (5.4). Поэтому из теоремы 5.1 следует знакорегулярность функции Грина $G(t, s)$ задачи (1.1)-(1.2). Кроме того, если $x \in \mathscr{D}_{1}$, то равенство $S(L x)=S(x)$, ввиду (6.5) и либо (6.11), (6.14), либо $(6.10),(6.15)$ возможно лишб при

$$
\operatorname{sign}_{1} x(a+) \operatorname{sign}_{1} L x(a+)=(-1)^{n-m},
$$

что ввиду второй части теоремы 5.1 означает, что $(-1)^{n-m} G(t, s)$ является осцилляционным ядром. Теорема 1.3 доказана полностью. 


\section{§7. Завершение доказательства теорем 1.1 и 1.2}

В данном параграфе завершается доказательство теорем 1.2 и 1.1. В соответствии с условиями этих теорем на протяжении всего параграфа по умолчанию считаем, что система $\left\{v_{i}\right\}_{1}^{n}$ является фундаментальной системой решений уравнения (1.6), у которой все вронскианы $v_{i j}(t)(i=\overline{0, m} ; j=\overline{0, n-m})$ в $(a, b)$ отличны от нуля и совпадают по знаку при совпадении порядков. Поэтому у этих вронскианов характеристики знаков $\sigma_{i j}$ не зависят от $t$ и совпадают при совпадении сумм индексов, на основании чего для этих характеристик будем также использовать обозначения с одним индексом, считая при $k=i+j$, что $\sigma_{k}=\sigma_{i+j}=\sigma_{i j}$. Далее, при $x \in \mathscr{D}_{1}$ наряду с введенными в $\S 6$ обозначениями $x_{i j}, \xi_{i j}^{a}, \xi_{i j}^{b}$ будем использовать обозначения

$$
\tilde{x}_{i j}(t)=\sigma_{i+j} \sigma_{i+j+1} x_{i j}(t), \quad \widetilde{\xi}_{i j}^{a}=\sigma_{i+j} \sigma_{i+j+1} \xi_{i j}^{a}, \quad \widetilde{\xi}_{i j}^{b}=\sigma_{i+j} \sigma_{i+j+1} \xi_{i j}^{b},
$$

где для соблюдения формальностей считаем $\sigma_{n+1}=\sigma_{n}$.

Сначала приведем одно вронскианное тождество общего характера.

Лемма 7.1. Пусть $m \geqslant 2$ и $z_{1}(t), z_{2}(t), \ldots, z_{k+m}(t)$ - достаточно гладкие функиии. Тогда

$$
\sum_{i=k+1}^{k+m}(-1)^{i}\left[z_{1}, \ldots, z_{k}, z_{i}\right](t)\left[z_{1}, \ldots, z_{i-1}, z_{i+1}, \ldots, z_{k+m}\right](t)=0 .
$$

ДокАЗАТЕЛЬСтво. Привлекая обозначения § 2 , вьпишем очевидные тождества

$$
\left[z_{1}, \ldots, z_{k}, z_{i}\right](t)=\sum_{j=0}^{k} z_{i}^{(j)}(t) z\left(\begin{array}{l}
\{0, \ldots, k\} \backslash j \\
\{1, \ldots, k\}
\end{array}\right)(t)(-1)^{j+k} \quad(i=\overline{1, k+m}) .
$$

Из этих тождеств следует, что в определителе

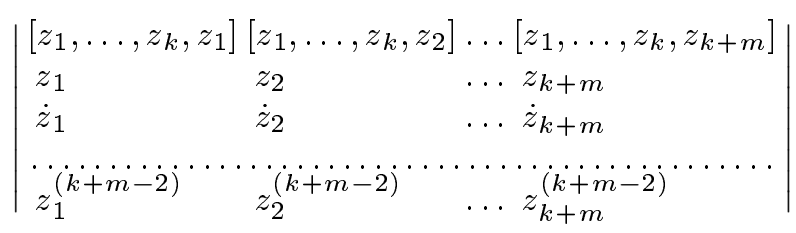

первая строка является линейной комбинацией следующих за ней $k+1$ строк, а значит, сам определитель равен нулю. Раскрывая определитель (7.3) по первой строке и учитывая, что первые $k$ элементов этой строки заведомо равны нулю, приходим к соотношению (7.2). Лемма доказана.

Отметим, что при $m=3$ соотношение (7.2) фактически является вронскианным аналогом известного детерминантного тождества Фекете (см. [4]) и применительно к нашей задаче с учетом обозначений (7.1) может быть переформулировано в следуюшем, удобном для дальнейшего использования, виде.

СлЕДСТВИЕ 7.1. Для любых $x(t) \in \mathscr{D}_{1}$ nрu $i=\overline{0, m-1}, j=\overline{0, n-m-1}$ справедливы соотношения

$$
\left|v_{i+1 j+1}(t)\right| \tilde{x}_{i j}(t)=\left|v_{i j+1}(t)\right| \tilde{x}_{i+1 j}(t)-\left|v_{i+1 j}(t)\right| \tilde{x}_{i j+1}(t) .
$$


ЛЕмма 7.2. Пусть у матрицы $\left\|\xi_{i j}\right\|(i=\overline{0, m}, j=\overline{0, n-m})$ все әлементь по модулю равнь единиче и удовлетворяют условиям

$$
S^{-}\left(\xi_{i j}, \xi_{i+1 j}\right) \leqslant S^{-}\left(\xi_{i j}, \xi_{i j+1}\right)
$$

npu

$$
i=\overline{0, m-1}, \quad j=\overline{0, n-m-1} \text {. }
$$

Тогда при условиях (7.6), $p=\overline{i+1, m}, r=\overline{j+1, n-m}$ справедливы соотношения

$$
\begin{aligned}
S^{-}\left(\xi_{i j}, \xi_{i+1 j}, \ldots, \xi_{p j}, \xi_{p j+1},\right. & \left.\ldots, \xi_{p r}\right) \\
& \leqslant S^{-}\left(\xi_{i j}, \xi_{i j+1}, \ldots, \xi_{i r}, \xi_{i+1 r}, \ldots, \xi_{p r}\right) .
\end{aligned}
$$

ДокАЗАТЕльство. Прежде всего заметим, что при (7.6) справедливы неравенства

$$
S^{-}\left(\xi_{i j}, \xi_{i+1 j}, \xi_{i+1 j+1}\right) \leqslant S^{-}\left(\xi_{i j}, \xi_{i j+1}, \xi_{i+1 j+1}\right) .
$$

Действительно, очевидно, что левая и правая части (7.8) могут быть неравньми лишь в случае, если $\xi_{i+1 j} \neq \xi_{i j+1}$ и $\xi_{i j}=\xi_{i+1 j+1}$ одновременно. В этом случае ввиду (7.5) $\xi_{i j}=\xi_{i+1 j}=-\xi_{i j+1}=\xi_{i+1 j+1}$, т.е. (7.8) заведомо выполняется.

Таким образом, (7.7) справедливо при (7.6), $p=i+1, r=j+1$. Исходя из этого, индукцией по $r$ легко показать, что (7.7) справедливо и при (7.6), $p=i+1$, $r=\overline{j+1, n-m}$. Действительно, если (7.7) имеет место при $p=i+1, r=l$, то

$$
S^{-}\left(\xi_{i j}, \xi_{i+1 j}, \xi_{i+1 j+1}, \ldots, \xi_{i+1 l}, \xi_{i+1 l+1}\right) \leqslant S^{-}\left(\xi_{i j}, \xi_{i j+1}, \ldots, \xi_{i l}, \xi_{i+1 l}, \xi_{i+1 l+1}\right)
$$

и ввиду (7.8)

$$
S^{-}\left(\xi_{i j}, \xi_{i j+1}, \ldots, \xi_{i l}, \xi_{i+1 l}, \xi_{i+1 l+1}\right) \leqslant S^{-}\left(\xi_{i j}, \xi_{i j+1}, \ldots, \xi_{i l}, \xi_{i l+1}, \xi_{i+1 l+1}\right)
$$

Поэтому (7.7) имеет место при $p=i+1, r=l+1$, а значит, и при (7.6), $p=i+1$, $r=\overline{j+1, n-m}$.

Очевидно, что на основании доказанного аналогичным образом, но уже индукцией по $p$ (7.7) проверяется и в общем случае. Лемма доказана.

Лемма 7.3. Пусть $x(t) \in \mathscr{D}_{1}$. Тогда

$$
\begin{aligned}
S^{-}\left(\widetilde{\xi}_{00}^{a}, \ldots, \widetilde{\xi}_{m 0}^{a}, \widetilde{\xi}_{m 1}^{a}, \ldots, \widetilde{\xi}_{m n-m}^{a}\right) \\
\quad \leqslant S^{-}\left(\widetilde{\xi}_{00}^{a}, \ldots, \widetilde{\xi}_{0 n-m}^{a}, \widetilde{\xi}_{1 n-m}^{a}, \ldots, \widetilde{\xi}_{m n-m}^{a}\right) \\
S^{-}\left(\widetilde{\xi}_{00}^{b}, \ldots, \widetilde{\xi}_{m 0}^{b}, \widetilde{\xi}_{m 1}^{b}, \ldots, \widetilde{\xi}_{m n-m}^{b}\right) \\
\quad \leqslant S^{-}\left(\widetilde{\xi}_{00}^{b}, \ldots, \widetilde{\xi}_{0 n-m}^{b}, \widetilde{\xi}_{1 n-m}^{b}, \ldots, \widetilde{\xi}_{m n-m}^{b}\right) .
\end{aligned}
$$


ДоКАЗАТЕЛЬСтво. Сразу отметим, что доказательство справедливости неравенств (7.9) и (7.10) сводится к проверке условий леммы 7.2 и ввиду полной аналогии рассуждений в обоих случаях мы ограничимся лишш обоснованием (7.9). Для этого, используя следствие 7.1, докажем, что при $i=\overline{0, m-1}, j=\overline{0, n-m-1}$ справедливы неравенства

$$
S^{-}\left(\widetilde{\xi}_{i j}^{a}, \widetilde{\xi}_{i+1 j}^{a}\right) \leqslant S^{-}\left(\widetilde{\xi}_{i j}^{a}, \widetilde{\xi}_{i j+1}^{a}\right)
$$

Так как $x(t) \in \mathscr{D}_{1}$, то при фиксированных $i, j$ найдется точка $d \in(a, b)$ такая, что на $(a, b)$ ни одна из функций $\tilde{x}_{i j}, \tilde{x}_{i+1 j}, \tilde{x}_{i j+1}$ не меняет знак, и хотя бы одно из значений

$$
\tilde{x}_{i j}(d), \tilde{x}_{i+1 j}(d), \tilde{x}_{i j+1}(d)
$$

отлично от нуля. Тогда ввиду (7.4) в (7.12) либо вообще нет нулей, либо имеется ровно один нуль. Кроме того, очевидно, что отличие от нуля любого элемента в (7.12) означает, что его знак совпадает со знаком соответствующей из величин $\widetilde{\xi}_{i j}^{a}, \widetilde{\xi}_{i+1 j}^{a}, \widetilde{\xi}_{i j+1}^{a}$. Поэтому из (7.4) следует, что ни в случае, когда в (7.12) нет нулей, ни в случае, когда в (7.12) ровно один нуль, не может выполняться равенство $\widetilde{\xi}_{i j}^{a}=-\widetilde{\xi}_{i+1 j}^{a}=\widetilde{\xi}_{i j+1}^{a}$, необходимое для нарушения (7.11).

Таким образом, неравенство (7.11) обязательно вьполняется, а значит, матрица $\left\|\widetilde{\xi}_{i j}^{a}\right\|(i=\overline{0, m}, j=\overline{0, n-m})$ удовлетворяет условиям леммы 7.2 , откуда следует справедливость (7.9). Лемма доказана.

Теперь можно завершить доказательство теоремы 1.2 .

Итак, пусть система функций $\left\{v_{i}(t)\right\}_{1}^{n}$ является фундаментальной системой решений уравнения (1.6), удовлетворяюшей условию (1.11), и, кроме того, все вронскианы $v_{i j}(t)$ в $(a, b)$ положительны. Тогда $\sigma_{k}=1(k=\overline{0, n})$. Поэтому при $x(t) \in \mathscr{D}_{1}$ все $\widetilde{\xi}_{i j}^{a}=\xi_{i j}^{a}, \widetilde{\xi}_{i j}^{b}=\xi_{i j}^{b}$ и из леммы 7.3 и неравенств $(6.10),(6.11)$ следует, что

$S^{-}\left(\xi_{00}^{a}, \ldots, \xi_{m 0}^{a}, \xi_{m 1}^{a}, \ldots, \xi_{m n-m}^{a}\right) \leqslant n-m \leqslant S^{-}\left(\xi_{00}^{b}, \ldots, \xi_{m 0}^{b}, \xi_{m 1}^{b}, \ldots, \xi_{m n-m}^{b}\right)$

В случае равенства левой и правой частей этого соотношения, очевидно, каждая из них равняется $n-m$ и $\xi_{m n-m}^{a}=(-1)^{n-m} \xi_{00}^{a}, \xi_{m n-m}^{b}=(-1)^{n-m} \xi_{00}^{b}$. С учетом этих соотношений из леммы 6.2 и теоремы 5.1 следует, что после умножения на $(-1)^{n-m}$ функция Грина задачи (1.1)-(1.2) становится осцилляционньм ядром. Таким образом, теорема 1.2 доказана полностью.

На основании теоремы 1.2 легко обосновать справедливость достаточности и для теоремы 1.1 в тех частных случаях, когда для фигурирующей в ней системы функций $\sigma_{k}=(-1)^{k(n-m)}$ при $k=\overline{0, n}$. Однако в общем случае, когда при $k \leqslant \min \{m, n-m\}$ знаки $\sigma_{k}$ чередуются произвольньм образом, доказательство достаточности для теоремы 1.1 сушественно усложняется. С целью преодоления возникающих здесь трудностей докажем следуюшее утверждение.

ЛЕмма 7.4. Пусть все әлементы векторов

$$
\eta=\left(\eta_{0}, \eta_{1}, \ldots, \eta_{n}\right), \zeta=\left(\zeta_{0}, \zeta_{1}, \ldots, \zeta_{n}\right), \theta=\left(\theta_{0}, \theta_{1}, \ldots, \theta_{n}\right)
$$


по модулю равны единище и для некоторого $r(0 \leqslant r \leqslant n)$ все $\theta_{k}=\theta_{n}$ при $k=\overline{r, n}$. Тогда если

$$
\begin{gathered}
\widetilde{\eta}_{k}=\theta_{k} \eta_{k} \quad(k=\overline{0, n}), \quad \widetilde{\eta}=\left(\widetilde{\eta}_{0}, \ldots, \widetilde{\eta}_{n}\right), \\
\widetilde{\zeta}_{k}=\theta_{k} \zeta_{k} \quad(k=\overline{0, n}), \quad \widetilde{\zeta}=\left(\widetilde{\zeta}_{0}, \ldots, \widetilde{\zeta}_{n}\right), \\
R_{1}=S^{-}(\widetilde{\eta})-S^{-}(\widetilde{\zeta})+2 S^{-}\left(\zeta_{r}, \ldots, \zeta_{n}\right), \\
R_{2}=S^{-}(\widetilde{\zeta})-S^{-}(\widetilde{\eta})+2 S^{-}\left(\eta_{r}, \ldots, \eta_{n}\right),
\end{gathered}
$$

$m o$

$$
\max \left\{R_{1}, R_{2}\right\} \leqslant S^{-}(\eta)+S^{-}(\zeta) \leqslant \min \left\{R_{1}, R_{2}\right\}+2 r
$$

ДокАЗАТЕльство. Прежде всего отметим, что ввиду равноправия векторов $\eta$ и $\zeta$ для доказательства (7.13) достаточно обосновать неравенство

$$
R_{1} \leqslant S^{-}(\eta)+S^{-}(\zeta) \leqslant R_{1}+2 r .
$$

Сначала докажем неравенство (7.14) для случая $r=n$, в котором оно принимает вид

$$
S^{-}(\widetilde{\eta})-S^{-}(\widetilde{\zeta}) \leqslant S^{-}(\eta)+S^{-}(\zeta) \leqslant S^{-}(\widetilde{\eta})-S^{-}(\widetilde{\zeta})+2 n .
$$

При $n=0$ это неравенство очевидно, а при $n=1$ следует из того, что в этом случае его левая, средняя и правая части соответственно имеют множествами своих значений $\{-1,0,1\},\{0,1,2\},\{1,2,3\}$ и совпадают между собой по четности. При $n>1$ соотношения (7.15) очевидным образом следуют по индукции с учетом равенств

$$
\begin{aligned}
& S^{-}(\eta)=S^{-}\left(\eta_{0}, \ldots, \eta_{n-1}\right)+S^{-}\left(\eta_{n-1}, \eta_{n}\right), \\
& S^{-}(\zeta)=S^{-}\left(\zeta_{0}, \ldots, \zeta_{n-1}\right)+S^{-}\left(\zeta_{n-1}, \zeta_{n}\right), \\
& S^{-}(\widetilde{\eta})=S^{-}\left(\widetilde{\eta}_{0}, \ldots, \widetilde{\eta}_{n-1}\right)+S^{-}\left(\widetilde{\eta}_{n-1}, \widetilde{\eta}_{n}\right), \\
& S^{-}(\widetilde{\zeta})=S^{-}\left(\widetilde{\zeta}_{0}, \ldots, \widetilde{\zeta}_{n-1}\right)+S^{-}\left(\widetilde{\zeta}_{n-1}, \widetilde{\zeta}_{n}\right),
\end{aligned}
$$

справедливость которых обеспечивается отличием от нуля всех элементов векторов.

Из справедливости (7.15) при $r=n$ следует, что при всех $r<n$ заведомо выполняются неравенства

$$
\begin{array}{r}
S^{-}\left(\widetilde{\eta}_{0}, \ldots, \widetilde{\eta}_{r}\right)-S^{-}\left(\widetilde{\zeta}_{0}, \ldots, \widetilde{\zeta}_{r}\right) \leqslant S^{-}\left(\eta_{0}, \ldots, \eta_{r}\right)+S^{-}\left(\zeta_{0}, \ldots, \zeta_{r}\right), \\
2 r+S^{-}\left(\widetilde{\eta}_{0}, \ldots, \widetilde{\eta}_{r}\right)-S^{-}\left(\widetilde{\zeta}_{0}, \ldots, \widetilde{\zeta}_{r}\right) \geqslant S^{-}\left(\eta_{0}, \ldots, \eta_{r}\right)+S^{-}\left(\zeta_{0}, \ldots, \zeta_{r}\right) .
\end{array}
$$

Кроме того, поскольку при $k \geqslant r$ все $\theta_{k}=\theta_{n}$, справедливо равенство

$$
S^{-}\left(\widetilde{\eta}_{r}, \ldots, \widetilde{\eta}_{n}\right)+S^{-}\left(\widetilde{\zeta}_{r}, \ldots, \widetilde{\zeta}_{n}\right)=S^{-}\left(\eta_{r}, \ldots, \eta_{n}\right)+S^{-}\left(\zeta_{r}, \ldots, \zeta_{n}\right) .
$$

Поочередно добавляя это равенство к неравенствам (7.16) и (7.17) и учитьвая, что $R_{1}=S^{-}(\widetilde{\eta})-S^{-}\left(\widetilde{\zeta}_{0}, \ldots, \widetilde{\zeta}_{r}\right)+S^{-}\left(\widetilde{\zeta}_{r}, \ldots, \widetilde{\zeta}_{n}\right)$, приходим к неравенству $(7.14)$. Лемма доказана.

В $\S 4$ было доказано, что если функция Грина краевой задачи (1.1)-(1.2) является знакорегулярной, то задача удовлетворяет условию (Б) из $\S 1$. Доказываемое далее обрашение этого утверждения является решаюшим моментом обоснования достаточности для теоремы 1.1 в общем случае. 
ТЕОРема 7.1. Пусть для краевой задачи (1.1)-(1.2) выполнено условие (Б). Тогда функиия Грина этой задачи является знакорегулярным ядром.

ДокАЗАТЕЛЬСтво. Для системы функций $\left\{v_{i}\right\}_{1}^{n}$, фигурируюшей в (Б), и произвольной функции $x(t) \in \mathscr{D}_{1}$ положим

$$
\begin{aligned}
\eta^{a} & =\left(\xi_{00}^{a}, \ldots, \xi_{m 0}^{a}, \xi_{m 1}^{a}, \ldots, \xi_{m n-m}^{a}\right), \\
\zeta^{a} & =\left(\xi_{00}^{a}, \ldots, \xi_{0 n-m}^{a}, \xi_{1 n-m}^{a}, \ldots, \xi_{m n-m}^{a}\right), \\
\widetilde{\eta}^{a} & =\left(\widetilde{\xi}_{00}^{a}, \ldots, \widetilde{\xi}_{m 0}^{a}, \widetilde{\xi}_{m 1}^{a}, \ldots, \widetilde{\xi}_{m n-m}^{a}\right), \\
\widetilde{\zeta}^{a} & =\left(\widetilde{\xi}_{00}^{a}, \ldots, \widetilde{\xi}_{0 n-m}^{a}, \widetilde{\xi}_{1 n-m}^{a}, \ldots, \widetilde{\xi}_{m n-m}^{a}\right), \\
\eta^{b} & =\left(\xi_{00}^{b}, \ldots, \xi_{m 0}^{b}, \xi_{m 1}^{b}, \ldots, \xi_{m n-m}^{b}\right), \\
\zeta^{b} & =\left(\xi_{00}^{b}, \ldots, \xi_{0 n-m}^{b}, \xi_{1 n-m}^{b}, \ldots, \xi_{m n-m}^{b}\right), \\
\widetilde{\eta}^{b} & =\left(\widetilde{\xi}_{00}^{b}, \ldots, \widetilde{\xi}_{m 0}^{b}, \widetilde{\xi}_{m 1}^{b}, \ldots, \widetilde{\xi}_{m n-m}^{b}\right), \\
\widetilde{\zeta}^{b} & =\left(\widetilde{\xi}_{00}^{b}, \ldots, \widetilde{\xi}_{0 n-m}^{b}, \widetilde{\xi}_{1 n-m}^{b}, \ldots, \widetilde{\xi}_{m n-m}^{b}\right)
\end{aligned}
$$

и покажем, что

$$
\begin{gathered}
S^{-}\left(\eta^{a}\right)+S^{-}\left(\zeta^{a}\right) \leqslant 2(n-m), \\
S^{-}\left(\eta^{b}\right)+S^{-}\left(\zeta^{b}\right) \geqslant 2(n-m) .
\end{gathered}
$$

Согласно условию (Б) при $r=n-m$ выполнены равенства $\sigma_{r}=\sigma_{r+1}=$ $\cdots=\sigma_{n}\left(=\sigma_{n+1}\right)$. Поэтому при $r=n-m$ к векторам $\eta^{a}, \zeta^{a}, \widetilde{\eta}^{a}, \widetilde{\zeta}^{a}$ и $\eta^{b}, \zeta^{b}$, $\widetilde{\eta}^{b}, \widetilde{\zeta}^{b}$ можно применить лемму 7.4, из которой следуют неравенства

$$
\begin{aligned}
& S^{-}\left(\eta^{a}\right)+S^{-}\left(\zeta^{a}\right) \leqslant S^{-}\left(\widetilde{\eta}^{a}\right)-S^{-}\left(\widetilde{\zeta}^{a}\right)+2 S^{-}\left(\xi_{0 n-m}^{a}, \ldots, \xi_{m n-m}^{a}\right)+2(n-m), \\
& S^{-}\left(\eta^{b}\right)+S^{-}\left(\zeta^{b}\right) \geqslant S^{-}\left(\widetilde{\zeta}^{b}\right)-S^{-}\left(\widetilde{\eta}^{b}\right)+2\left(\xi_{m 0}^{b}, \xi_{m 1}^{b}, \ldots, \xi_{m n-m}^{b}\right) .
\end{aligned}
$$

Кроме того, условие (Б) обеспечивает для системы $\left\{v_{i}\right\}_{1}^{n}$ справедливость следствия 6.1 и леммы 7.3. Из следствия 6.1 , в частности, следуют равенства

$$
\begin{gathered}
S^{-}\left(\xi_{0 n-m}^{a}, \xi_{1 n-m}^{a}, \ldots, \xi_{m n-m}^{a}\right)=0, \\
S^{-}\left(\xi_{m 0}^{b}, \xi_{m 1}^{b}, \ldots, \xi_{m n-m}^{b}\right)=n-m,
\end{gathered}
$$

а из леммы 7.3 следуют неравенства

$$
S^{-}\left(\widetilde{\eta}^{a}\right) \leqslant S^{-}\left(\widetilde{\zeta}^{a}\right), \quad S^{-}\left(\widetilde{\eta}^{b}\right) \leqslant S^{-}\left(\widetilde{\zeta}^{b}\right) .
$$

С учетом (7.22) и (7.23) из (7.20) следует (7.18), а из (7.21) следует (7.19).

Из (7.18) и (7.19) следует справедливость по крайней мере одного из неравенств

$$
S^{-}\left(\eta^{a}\right) \leqslant S^{-}\left(\eta^{b}\right), \quad S^{-}\left(\zeta^{a}\right) \leqslant S^{-}\left(\zeta^{b}\right) .
$$

Любое из них ввиду леммы 6.2 означает, что $S(L x) \geqslant S(x)$. Отсюда по теореме 5.1 следует, что функция Грина рассматриваемой задачи является знакорегулярным ядром. Теорема доказана.

Теперь можно завершить доказательство теоремы 1.1. 
Итак, пусть система функций $\left\{v_{i}(t)\right\}_{1}^{n}$ является фундаментальной системой решений уравнения (1.6), определяемой условиями (1.10), и все вронскианы $v_{i j}(t)$ в $(a, b)$ отличны от нуля и совпадают по знаку при совпадении порядков. Тогда ввиду следствия 2.1 при $i+j \geqslant \max \{m, n-m\}$ эти вронскианы будут совпадать по знаку с $(-1)^{(n-i-j)(n-m)} v_{m n-m}(t)$. В таком случае задача $(1.1)-(1.2)$ удовлетворяет условию (Б), так как теперь очевидно, что фигурирующая в (Б) система может быть получена из $\left\{v_{i}\right\}_{1}^{n}$ умножением каждой функции системы на $(-1)^{n-m}$. Но по теореме 7.1 выполнение условия (Б) означает знакорегулярность функции Грина рассматриваемой задачи, что завершает доказательство достаточности в теореме 1.1. Напомним, что необходимость в этой теореме и вторая ее часть были доказаны в $\S 4$. Таким образом, теорема 1.1 доказана полностью.

Автор выражает благодарность А. Ю. Левину за обсуждение статьи, способствовавшее ее улучшению. На завершаюшей стадии работы над статьей автор пользовался поддержкой программы "Университеты России".

\section{Список литературы}

1. Степанов Г. Д. Критерий осцилляционности функции Грина двухточечной краевой задачи // Докл. АН СССР. 1973. Т. 213. № 4. С. 793-794.

2. Степанов Г.Д. Критерий знакорегулярности функции Грина двухточечной краевой задачи // Докл. АН СССР. 1977. Т. 234. № 4. С. 765-767.

3. Левин А. Ю., Степанов Г. Д. Одномерные краевые задачи с операторами, не понижающими числа перемен знака // Сиб. матем. журн. 1976. Т. 17. № 3. С. 606-626; Т. 17. № 4. C. $813-830$.

4. Гантмахер Ф.P., Крейн М.Г. Осцилляционные матрищы и ядра и малые колебания механических систем. М.-Л.: Гостехиздат, 1950.

5. Крейн М. Г., Финкельштейн Г. М. О вполне неотрицательных функциях Грина обыкновенных дифференциальных операторов // Докл. АН СССР. 1939. Т. 24 . №3. C. $220-223$.

6. Крейн М. Г. О несимметрических осцилляционных функциях Грина обыкновенных дифференцальных операторов // Докл. АН СССР. 1939. Т. 25. № 8. С. 643-646.

7. Kellogg O. D. The Oscillation of Functions of an Orthogonal Set // Amer. J. Math. 1916. V. 38. P. 1-5.

8. Kellogg O. D. Orthogonal Function Sets Arising from integral Equations // Amer. J. Math. 1918. V. 40. P. 145-154.

9. Kellogg O.D. Interpolation properties of orthogonal sets of solutions of differential equations // Amer. J. Math. 1918. № 40. P. 220-234.

10. Гантмахер Ф. Р., Крейн М. Г. Об одном классе детерминантов в связи с интегральными ядрами Келлога // Матем. сб. 1933. Т. 40. С. 501-508.

11. Гантмахер Ф.Р., Крейн М. Г. Sur les matrices oscillatoires et completement non negatives // Compositio Mathematica. 1937. V. 4. P. 445-476.

12. Гантмахер Ф. Р. О несимметрических ядрах Келлога // Докл. АН СССР. 1936. Т. 10. № 1. C. 3-5.

13. Крейн М. Г., Нудельман А. А. Проблема моментов Маркова и экстремальные задачи. M.: Наука, 1973.

14. Karlin S. Total Positivity. Stanford: Stanford Univ. Press, 1968.

15. Карлин $C$., Cтадден $B$. Чебышевские системы и их применение в анализе и статистике. M.: Наука, 1976.

16. Баржовский Ю. С., Юдович В. И. Рождение вихрей Тейлора в случае разновращающихся цилиндров и спектральные свойства одного класса краевых задач // Докл. АН CCCP. 1978. T. 242. № 4. C. 784-787.

17. Макин P.C. О спектре стационарного односкоростного уравнения переноса с изотропным рассеянием // Докл. АН СССР. 1984. Т. 274. № 3. С. 536-540.

18. Polia $G$. On the meanvalue theorem corresponding to a given linear homogeneous differential equation // Trans. Amer. Math. Soc. 1922. V. 24. P. 312-324. 
19. Левин $A$. Ю. Неосцилляция решений уравнения $x^{(n)}+p_{1}(t) x^{(n-1)}+\cdots+p_{n}(t) x=0$ // УМН. 1969. Т. 24. № 2. С. 43-96.

20. Калафати П. Д. О функциях Грина обыкновенных дифференциальных уравнений // Докл. АН СССР. 1940. Т. 26. №6. С. 535-539.

21. Karon J. M. The sihn-regularity properties of a class of Green's, functions for ordinary differential equations // J. Differential Equations. 1969. № 6. P. 484-502.

22. Karlin S. Total Positivity, Interpolation by Splines and Green's, functions for ordinary differential equations // J. Approx. Theory. 1971. V. 4. №1. P. 91-112.

23. Schoenberg I. J. Über variationsverminderndelineare Transformationen // Math. Z. 1930. №32. P. 321-328.

24. Левин А. Ю., Степанов Г. Д. Одномерные краевые задачи с операторами, не понижающими числа перемен знака // УМН. 1975. Т. 30. № 1. С. 245-246.

25. Степанов Г. Д. О вещественности собственных значений некоторых краевых задач // Труды матем. фак-та ВГУ. № 7. Воронеж, 1972. С. 94-95.

26. Барковский Ю. С. Гипоосцилляционные и гиперосцилляционные дифференциальные операторы // Докл. АН СССР. 1982. Т. 267. № 2. С. 269-271.

27. Юдов ич В. И. Спектральные свойства осцилляционного дифференциального оператора на прямой // УМН. 1983. Т. 38. №1. С. 205-206.

28. Архипов В. П., Соболев А.В. Осцилляционные свойства вырождающихся дифференциальных операторов второго порядка // Докл. АН СССР. 1984. Т. 274. №4. С. 777-779.

29. Покорный Ю. В., Лазарев К. П. Некоторые осцилляционные теоремы для многоточечных задач // Дифференц. уравнения. 1987. Т. 23. № 4. С. 658-670.

30. Левин А. Ю. Абсолютная неосцилляционная устойчивость и смежные вопросы // Алгебра и анализ. 1992. Т. 4. №1. С. 154-166.

31. Боровских А. В., Покорный Ю. В. Системы Чебышёва-Хаара в теории разрьвных ядер Келлога // УМН. 1994. Т. 49. № 3. С. 3-42.

32. Боровских А.В., Лазарев К. П., Покорный Ю. В. Об осцилляционных спектральных свойствах разрывных краевых задач // Докл. АН. 1994. Т. 335. № 4. С. 409-412.

33. Калафати П. Д. Осцилляционные свойства фундаментальных функций краевых задач третьего порядка // Докл. АН СССР. 1962. Т. 143. № 3. С. 518-521.

34. Степанов Г. Д. Об эффективных критериях осцилляционности и знакорегулярности функций Грина двухточечной краевой задачи // Весенняя Воронежская математическая школа "Понтрягинские чтения-IV". Воронеж, 1993. С. 176.

35. Степанов Г. Д. О знакопостоянных класса $p$ матрицах и ядрах // Вестник Ярославского ун-та. 1973. № 5. С. 138-151.

36. Степанов Г. Д. Знакорегулярность и одномерные краевые задачи // Дис. ... канд. физ.-матем. наук. Ярославль, 1974.

37. Гантмахер Ф. Р. Теория матриц. М.: Наука, 1966.

38. Полиа Г., Сеге Г. Задачи и теоремы из анализа. Т. 2. М.: Гостехиздат, 1956.

Воронежский государственный педагогический университет

Поступила в редакцию 17.10 .1996 